\title{
Biogeografía analítica de la pteridoflora del Arco de Alborán: consecuencias para su status de protección
}

\author{
Ángel Enrique Salvo Tierra ${ }^{1}$, Ignacio J. Melero-Jiménez ${ }^{1}$, José García-Sánchez ${ }^{1}$, José Carlos Báez² \& \\ Antonio Flores-Moya ${ }^{1}$ \\ ${ }^{1}$ Departamento de Botánica y Fisiología Vegetal, Facultad de Ciencias, Universidad de Málaga, Campus de Teatinos, \\ Bulevar Louis Pasteur, 29010 Málaga \\ ${ }^{2}$ Instituto Español de Oceanografía, Centro Oceanográfico de Málaga (sede Fuengirola), 29640 Fuengirola (España). \\ Facultad de Ciencias de la Salud, Universidad Autónoma de Chile, Santiago de Chile, Chile.
}

\section{Correspondencia}

Á.E. Salvo-Tierra

e-mail: salvo@uma.es

Recibido: 3 febrero 2020

Aceptado: 14 abril 2020

Publicado on-line: 29 junio 2020

Editado por: Antonio Galán de Mera

\begin{abstract}
Resumen
Se ha analizado la pteridoflora del Arco de Alborán mediante la aplicación de índices pteridoflorísticos y su correlación con variables ambientales. Asimismo se han realizado análisis de agrupamiento de taxones para definir corotipos y de componentes principales para delimitar unidades pteridogeográficas. Con todos estos datos se realiza un análisis de conservación de taxones amenazados y se valora desde un punto de vista territorial la posible declaración del área de estudio con alguna figura de protección especial.
\end{abstract}

Palabras clave: Pteridofitas, Mediterráneo Occidental, Corotipos, Unidades fitogeográficas, Conservación de especies.

\section{Abstract}

Analytical biogeography of the pteridoflora of the Alboran Arc.

The pteridoflora of the Alboran Arc has been analyzed by applying pteridofloristic indices and its correlation with environmental variables. Likewise, taxa grouping analyzes have been carried out to define chorotypes and principal components analysis to delimit pteridogeographic units. With all these data, a conservation analysis of threatened taxa is carried out and the possible declaration of the study area with some special protection figure is assessed from a territorial point of view.

Key words: Pteridophytes, Western Mediterranean, Chorotypes, Phytogeographic units, Species conservation.

\section{Introducción}

El conocimiento básico y el monitoreo de la diversidad vegetal es el punto basal para la planificación del uso y manejo de los recursos, así como para la aplicación de las medidas de conservación, aprovechamiento, mitigación y restauración más adecuadas. Por su antigüedad las pteridofitas presentan áreas de distribución que son consecuencia de importantes cambios geológicos y climáticos. En la actualidad se reconocen 13.000 especies, en su gran mayoría distribuidas en la franja intertropical, quedando en las zonas extratropicales especies en su mayor parte de areales reducidos (Tryon \& Tryon, 1982; Given, 1993). Éstas presentan áreas más permanentes como resultante de una drástica selección debido a la combinación de la influencia de diferentes eventos ambientales y de la capacidad adaptativa de cada especie (Pichi-Sermolli, 1979). Precisamente esta es la razón de la importancia del análisis biogeográfico de las pteridofloras. Conociendo la distribución actual de las especies de pteridofitas en un espacio determinado es posible aproximarse a cuál ha sido el papel de la participación de los diferentes acontecimientos históricos sobre dicho territorio. En general, aquellas especies con un patrón de distribución similar suelen tener exigencias hídricas (fundamental para la reproducción sexual) y bioclimáticas similares (Pichi-Sermolli et al., 1988; Márquez et al., 1997). Su conocimiento posibilita, por extensión, comprender las condiciones ambientales de sus áreas de distribución actual, pasada y por ende prospectar escenarios futuros (Salvo \& GarcíaVerdugo, 1990).

En solo unos años la extinción de linajes de seres vivos ha pasado de ser una abstracción geológica a una realidad a la escala temporal humana en la época actual (Given, 1993). Las tasas actuales de extinción son difíciles de estimar especialmente en grupos taxonómicos que están mal documentados, pero se ha argumentado que puede haber un aumento de varios órdenes de magnitud a lo largo de este siglo para generar la mayor extinción masiva durante al menos los últimos 65 millones de años (Badii et al., 2015). En la actualidad el rápido calentamiento global, debido a la actividad humana (Oreskes, 2004), es previsible que afecte a ecosistemas tan sensibles como los del Arco de Alborán. Muchos autores prevén que entre la biota existirán ganadores (especies generalistas de ambientes templado-cálidas) y perdedores (especies de ambientes circumpolares o 
de topoclimas excepcionales) (Coll et al., 2013). Por este motivo es importante caracterizar la distribución biogeográfica de las especies, principalmente de aquellas de carácter estenoico. Recientemente ha sido realizada una revisión de la utilidad de los pteridofitas como bioindicadores (Della \& Falkenberg, 2019) reconociendo el uso potencial creciente, entre otros de la calidad ambiental, la contaminación o el cambio climático.

El objetivo del presente artículo es estudiar desde una perspectiva de Biogeografía analítica las variables ecogeográficas que determinan la distribución de la diversidad de la pteridoflora en el Arco de Alborán.

\section{Material y método}

\section{Área de estudio}

El Arco de Alborán (conocido geológicamente como Arco de Gibraltar) está formado por una cadena montañosa formada por la Cordillera Bética, el estrecho de Gibraltar y el Rif (Fig. 1). Su origen geológico surge por la aproximación de la placa tectónica africana y la microplaca ibérica durante el terciario (65 Ma) (Flinch, 1993). Tal aproximación jugó un papel fundamental para el intercambio biológico entre los dos continentes durante el Messiniense (5 $\mathrm{Ma}$ ) al establecerse puentes de comunicación por la desecación del Mediterráneo, hito de excepcional importancia por el cambio profundo de la composición florística y de vegetación (Sadori et al., 2013). Estos eventos geológicos, y sus consecuencias climáticas, han sido los responsables de la importante diversidad florística y fitocenológica del Arco Alboránico (Medail \& Quézel, 1999), con un alto número de relictos, desde los paleotropicales lauroides cuya presencia es previa a la crisis messiniense, a los iranoturanianos incorporados durante esa crisis o a los acantonamientos glaciales (Pichi-Sermolli, 1979, Allen, 2003).

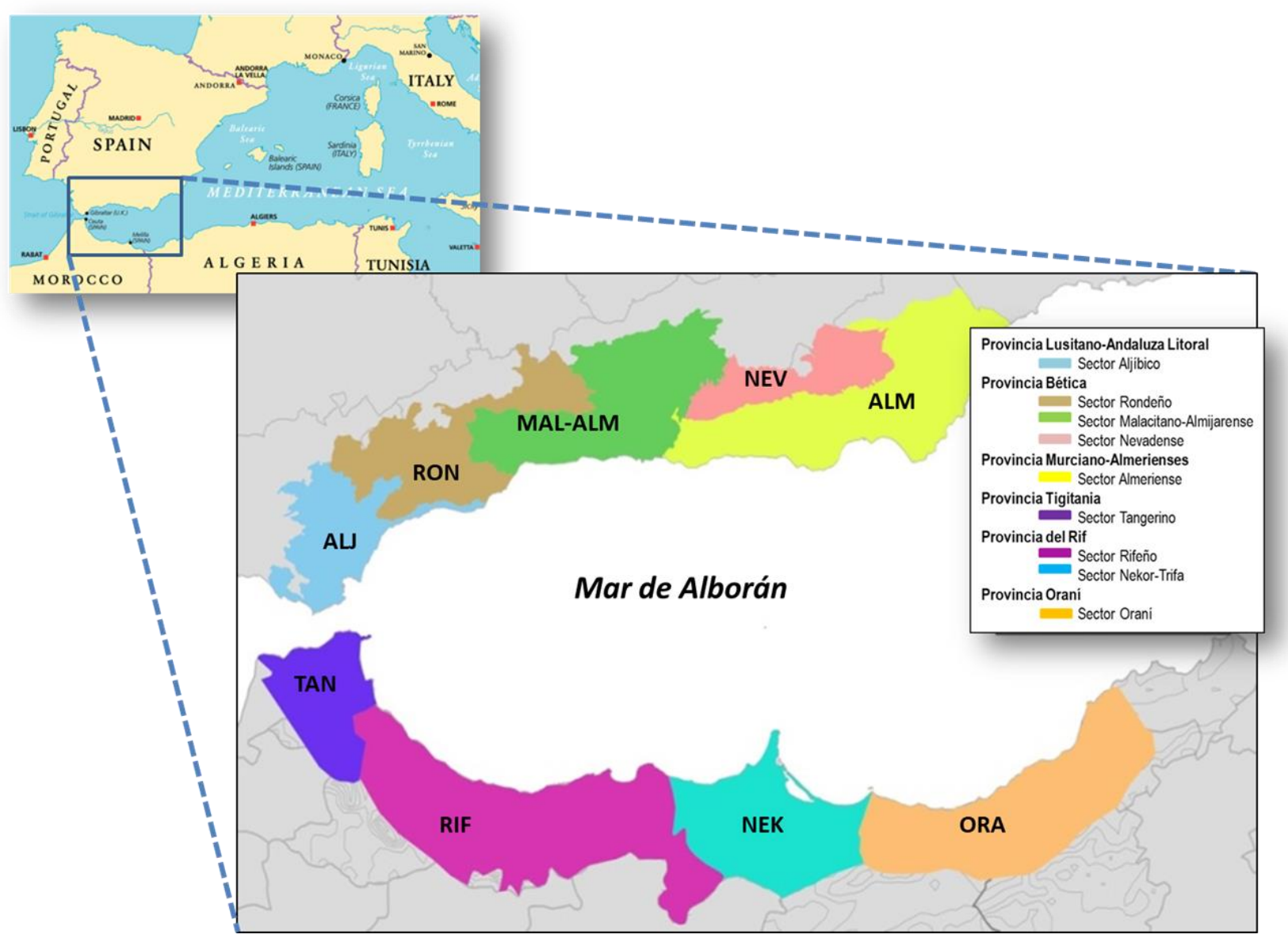

Figura 1. Localización y sectorización biogeográfica del Arco de Alborán.

Figure 1. Biogeographic location and sectorization of the Alboran Arc.

\section{Determinación de las UGOs}

En consecuencia se ha procedido a definir las Unidades Geográficas Operativas (en adelante UGOs) en base a los siguientes criterios: 1) El Arco de Alborán está dividido principalmente en tres unidades metamórficas, de carácter alóctono, procedentes de la placa Mesomediterránea: a) la unidad inferior corresponde al Complejo NevadoFilábride; b) sobre ella se dispone el Complejo Alpujárride, formado por materiales con indicios de un metamorfismo de bajo grado, desde facies de esquistos azules en las partes inferiores hasta alcanzar la facies de eclogita y el desarrollo de las peridotitas de Ronda en las partes superiores; c) el 
Complejo Maláguide ocupa la posición más alta, de manera que recubre a los materiales de los complejos anteriores y apenas muestra un metamorfismo alpino, solo en algunas regiones más profundas y de muy bajo grado.

En cuanto a las unidades sedimentarias, destacan los afloramientos neógenos de Flysch sobre las cordilleras Subbéticas y el Rif, en ambos casos en la zona más occidental (Flinch, 1993).

2) Si bien el piso bioclimático dominante es el termomediterráneo, en determinadas áreas litorales se aprecian situaciones características del inframediterráneo (Rivas-Martínez, 2007; RivasMartínez \& Rivas-Sáenz, 2019). Este piso inferior es de reciente observación en especial en la vertiente europea, lo que supone una manifestación de un cambio climático favorecido por el aumento de temperaturas medias y disminución de precipitaciones. En los complejos serranos y dependiendo de la altitud de los mismos tienen presencia los pisos mesomediterráneo, supramediterráneo, oromediterráneo y crioromediterráneo. En cuanto a los ombrotipos en el Arco de Alborán se observa un gradiente de W a E, variando desde hiperhúmedos y húmedos en la parte occidental hasta secos y semiáridos en las localidades orientales (Rivas-Martínez \& RivasSáenz, 2019; Valle-Tendero et al., 2004). 3) Desde un punto de vista biogeográfico el conjunto territorial se sitúa en la Región Mediterránea, estando representadas seis provincias que incluyen nueve sectores (Maire, 1952; Rivas-Martínez et al., 2014, 2017; Benabid \& Fennane, 1994; Rankou et al., 2013).

La determinación de las UGOs se ha realizado atendiendo a criterios de homogeneidad geofísica y en correspondencia con las unidades biogeográficas. Se han seleccionado nueve complejos serranos, uno por cada sector biogeográfico, en los que estuvieran representados el máximo de pisos bioclimáticos y ombrotipos: Gata, Nevada, Tejeda, Ronda, Aljibe, Tingitania, Chauen, Gurugú y Tlemcen. Las variables ambientales que caracterizan cada UGO y que son determinantes en desarrollo vital de su pteridoflora, se recogen en el Anexo 1. Se ha elegido una estación representativa en cada caso en virtud de la mayor diversidad pteridoflorística según las fuentes bibliográficas consultadas (ver el siguiente apartado). Las coordenadas de cada estación y la altitud máxima en cada UGO han servido para determinar mediante New_LocClim_1.10 (FAO, 2006) los valores de cuatro variables climáticas referidos a sus medias anuales (Tmed: temperatura media; Tmin: temperatura mínima; OSC: oscilación térmica, media de las diferencias diarias entre Tmax y Tmin; PLUV: precipitación total anual).

\section{Matriz básica de datos (MBD)}

Se ha elaborado un catálogo (Anexo 2) adaptado a la sistemática consensuada por el Pteridophyte Phylogeny Group PPG1 (2016). Se ha actualizado la base de datos AXIS (Salvo ined.) para la elaboración de las fichas pteridológicas de cada uno de los taxones. Para la conformación de las pteridofloras de cada una de las UGOs se han tenido en cuenta los registros de la colección MGC-Cormof del herbario de la Universidad de Málaga, además de la revisión bibliográfica referida a cada uno de los Sectores del área de estudio (Díez-Garretas \& Salvo-Tierra, 1980; Nieto et al., 1987; Salvo, 1982, 1994; Salvo \& Escámez, 1989; Varo \& Salvo, 1982), así como las síntesis corológicas recientes de Moreno-Saiz et al. (2017) para España, Fennane (2016) para Marruecos y Medjahdi et al. (2013) para Argelia. Se ha construido una matriz básica de datos (MBD) de presencia/ausencia de los 71 taxones para cada una de las UGOs (Anexo 3).

\section{Caracterización pteridogeográfica de las UGOs}

Para el análisis biogeográfico se ha seguido el método propuesto por Salvo \& García-Verdugo (1990) de la biogeografía numérica aplicada a la pteridología, basado en la composición pteridofítica de las UGOs. Dicha información fue procesada mediante el software PAST 3.25 (Hammer, 2019) y Excel 2016 v.16, y se calcularon diferentes índices pteridoflorísticos para la caracterización fitocenológica y epiontológica de cada UGO. Así se determinaron:

Riqueza pteridofítica. Además del número de taxones por UGO, se ha deducido el grado de rareza de cada taxón en el área estudiada, obtenido mediante el cálculo de la inversa de la media de los valores de presencia estandarizados, estableciendo 4 categorías por cuartiles: muy raro $(>1,22 ; 32,4 \%)$, raro $(0,79-1,22 ; 22,5 \%)$, ocasional $(0,51-0,79$; $23,9 \%)$, y frecuente $(<0,51 ; 21,1 \%)$.

Heterogeneidad. Se ha calculado la heterogeneidad específica $(\mathrm{H})$ de cada una de las nueve UGOs, para lo que se aplicó el Índice de Shannon (Shannon \& Weaver, 1949; Pla, 2006).

Ratio especie/género (S/G) y coeficiente genérico. El índice $S / G$ es un estimador del grado de antigüedad de la pteridoflora en las diferentes UGOs, así un valor próximo a la unidad indica la presencia de posibles relictualismos, mientras el alejamiento de la unidad supone una mayor capacidad de taxones infragenéricos (Palmgren, 1925; Jaccard, 1940; Järvinen, 1982; Eşanu 2018; Krug et al., 2008;). Además se ha calculado el coeficiente genérico, definido como la sumatoria de la proporción de especies por género en cada UGO.

Ratio entre especies con esporas monoletas respecto a triletas $(\mathrm{m} / \mathrm{t})$. Índice propuesto por Ito (1972) basado en la relación de especies de helechos con esporas monolaesuradas (monoletas) respecto a las trilaesuradas (triletas). En base a su observación en 64 pteridofloras, Ito (1978) propuso que: a) el cociente $\mathrm{m} / \mathrm{t}$ disminuye de $\mathrm{N} \mathrm{a} \mathrm{S}$; b) a igual latitud, las regiones más áridas presentan un valor menor; y c) a igual latitud el índice aumenta proporcionalmente con la altitud. Los datos se han obtenido de Salvo (1982, 1990).

Número cromosómico y grado de ploidía. Se han tenido en cuenta los datos registrados en la base de datos AXIS (Salvo ined.).

Espectro biológico y fitocenológico. El espectro biológico se basa en la determinación de los 
porcentajes de aparición de los biotipos (según la clasificación de Raunkier, revisado por Cornelissen et al., 2003) de los taxones. En cuanto al espectro fitocenológico se han estimado los porcentajes de representación de los hábitats preferentes en base a los taxones presentes en cada UGO según la sistemática propuesta por Salvo \& Garcia-Verdugo (1990).

Espectro de tracks biogeográficos. Consiste en un análisis basado en los 'tracks' generalizados (Croizat, 1958; Suc, 1984; Palamarev, 1989; Sluys, 1994; Thompson 2005; Fernández-Badillo et al., 2014) de taxones propuestos por Pichi-Sermolli (1979) y PichiSermolli et al. (1988), considerando su distribución actual y el posible origen de los mismos.

\section{Análisis de agrupamiento de UGOs}

A partir de la determinación de los valores de estos índices se ha procedido mediante el software PAST 3.25 (Hammer 2019) a la realización de análisis comparativos entre UGOs:

Jerarquización de UGOs y establecimiento de unidades pteridogeográficas (UPs). Para la definición de las UPs se ha realizado en primer lugar una doble estandarización de la MBD (Beals, 1984). Tras multiplicar el grado de rareza de cada especie sobre los valores de las mismas en la MBD estandarizada se ha calculado el índice de similitud por el método de Bray \& Curtis (1957) y se ha elaborado un dendrograma de agrupación con el algoritmo UPGMA. Mediante un análisis de componentes principales se han contrastado dichos resultados, considerando tanto las variables ambientales como los índices pteridoflorísticos.

Fronteras y flujos. Se han analizado las fronteras y flujos (Hernández-Bermejo \& Sainz de Ollero, 1983) en términos de conectividad potencial entre pares de UPs. Para ello, se ha elaborado una Matriz de Índices de Similitud mediante el coeficiente de Sørensen \& Dice (Dice 1945, Sørensen, 1957). Los valores obtenidos se han clasificado en 4 categorías de fronteras en virtud de los cuartiles de los valores más elevados: muy fuerte $(\leq 60 \%)$, fuerte $(61-70 \%)$, moderada $(71-80 \%)$ y débil $(>80 \%)$.

\section{Análisis de correspondencia entre variables ecogeográficas y UGOs}

Se ha realizado un análisis de la correlación entre cada variable ambiental y cada índice pteridoflorístico para las UGOs estudiadas.

\section{Caracterización de corotipos}

Para la determinación de corotipos se ha usado el algoritmo de la k-media (algoritmo de Lloyd), método de agrupamiento que tiene como objeto la partición de un conjunto de $n$ observaciones en $\mathrm{k}$ grupos en el que cada observación pertenece al grupo cuyo valor medio es más cercano (Likas et al., 2003).

\section{Resultados y discusión}

\section{Caracterización pteridogeográfica de las UGOs}

En el Anexo 4 se presenta el cuadro resumen con los valores de los índices pteridogeográficos estimados para cada UGO.

Riqueza pteridofítica. La pteridoflora del Arco de Alborán está integrada por 71 especies, variando entre 23 y 46 en las distintas UGOs (Tryon \& Tryon, 1982; Given, 1993). El Arco de Alborán se trataría de un centro secundario de diversidad de pteridofitas en regiones semi-xéricas y xéricas (Given, 1993), con considerables casos de adaptaciones morfológicas y reproductoras para hacer frente a las condiciones comúnmente consideradas antagonistas de las pteridofitas, particularmente el déficit hídrico estacional más o menos prolongado. Estas adaptaciones incluyen latencia estacional con desprendimiento de frondes, protección mediante escamas y numerosas bases de pecíolos (Kornas, 1985). Helechos como Asplenium hispanicum (Coss.) Greuter \& Burdet, A. ceterach L., Cosentinia vellea (Aiton) Tod., Paragymnopteris marantae (L.) K.H. Shing o las especies del género Oeosporangium constituyen un buen ejemplo de este tipo de adaptaciones. En el Anexo 5 se recoge el grado de rareza de cada taxón dentro del área de estudio, de los que 23 tendrían la consideración de muy raros y 16 raros.

Heterogeneidad. La heterogeneidad resultante tiene un promedio de 3,44, oscilando desde 3,05 en Gurugú hasta 3,74 en Aljíbe y Nevada. Se detectó una correlación altamente significativa entre este índice y el número de especies $(R=0,99)$, quedando fuera del $95 \%$ las unidades de Ronda y Tingitania.

Ratio $S / G$. El promedio del número de especies por género representado en cada pteridoflora es de 1,99, oscilando entre el 1,58 de la Tingitania y el 2,56 de Nevada. La correlación entre este cociente y el coeficiente genérico muestras anomalía en la unidad Aljibe, con un coeficiente genérico muy por encima del que le correspondería, y las más orientales de Gata y Tlemcen con valores del coeficiente por debajo del que le permitiría ajustarse a un nivel de confianza superior al $95 \%$.

Ratio $\mathrm{m} / \mathrm{t}$. Los resultados del índice $\mathrm{m} / \mathrm{t}$ obtenidos en las UGOs del Arco de Alborán varían desde 0,63 en la zona de Chauen hasta 1,75 en Tejeda. De los tres patrones señalados por Ito (1978), tan solo la altitud presenta una correlación significativa $(R=0,85)$, quedando fuera del $95 \%$ solo la unidad Tejeda, que presenta un valor superior al que le correspondería por su altitud máxima.

Número cromosómico y grado de ploidía. La antigüedad y ciclo biológico de las pteridofitas han conllevado a que presenten números cromosómicos muy elevados con una media muy superior a la de las plantas con flor. De hecho, si en las angiospermas la línea divisoria entre los números de cromosomas diploides y poliploides se ha establecido en $n=13$ ó 14 (Grant, 1981), se puede considerar que aproximadamente el $50 \%$ son poliploides, mientras que en pteridofitas el valor excede el $95 \%$. Estos valores sugieren que, en especial las polipodiópsidas, constituyen un grupo singular que exige una consideración especial al desarrollar ideas sobre 
patrones y procesos de evolución (Haufler 2002), en donde la ploidización (tanto autoploidía como aloploidía) han jugado un papel fundamental. Recientemente Rice et al. (2019) han puesto en evidencia que existe una clara tendencia latitudinal, con una frecuencia de poliploides que se aleja del Ecuador. El bioclima, especialmente la temperatura, resulta ser el predictor más influyente de la distribución de poliploides. Sin embargo, este efecto parece ser indirecto, estando mediado por la variación en las formas de vida de las plantas y, en menor medida, por la composición taxonómica y la riqueza de especies. Así la aparición de poliploides requiere de suficiente tiempo (es decir, perennialidad) y espacio (baja riqueza de especies) para competir con parientes diploides preadaptados.

El número cromosómico medio de la pteridoflora del Arco de Alborán es de 122 en la fase esporofítica, oscilando entre los 102 de la unidad tingitana y 141 en la nevadense. En cuanto al grado medio de ploidía es de 3 , e igualmente varía desde los 2,5 de Tingitania hasta los 3,2 de Nevada. Si bien parecería obvia una relación entre ambas variables, sin embargo ésta presenta un grado de correlación del $68 \%$, quedando fuera del intervalo de confianza del 95\% las pteridofloras de Gurugú y Gata.

Espectro biológico y fitocenológico. Existe un claro predominio de las especies hemicriptófitas, con un porcentaje promedio de $71 \%$, seguido por geófitos con una media de $21 \%$. Respecto al espectro fitocenológico existe un dominio de taxones pertenecientes a comunidades silicícolas de fisuras de rocas y muros no rezumantes de los pisos inferiores con un promedio de $30,3 \%$, seguido de aquellas comunidades que colonizan fisuras de rocas y muros calcícolas no rezumantes, tanto termófilas con un 15,8 \% como no termófilas con un $11,9 \%$.

Espectro de tracks biogeográficos. Se observa un reparto relativamente homogéneo de especies heterocóricas, latemediterráneas y submediterráneas, con un promedio superior al $20 \%$ cada tipología. A pesar de tener un porcentaje de distribución relativamente bajo, cabe destacar los endemismos mediterráneos y los relictos paleomediterráneos (alrededor del 7\% de media), al tratarse de excelentes indicadores de calidad ambiental y relictualismo.

Jerarquización de UGO y establecimiento de unidades pteridogeográficas. Los resultados del cálculo del índice de similitud se han representado en un dendrograma de agrupación (Fig. 2) con un coeficiente de correlación del $88,8 \%$. Al trazar una línea de fenon en un grado de similitud del $88 \%$ se desagregan cuatro agrupaciones: 1) Aljibe, 2) Tejeda y Nevada, 3) Chauen y Ronda y 4) el resto de unidades, que a una similitud del $91 \%$ se desagrega en dos subgrupos: $4 a$ ) Tingitania, y 4b) Gata, Gurugú y Tlemcen. Se conforman así 5 unidades pteridogeográficas (UP) (Fig. 3): UP 1 - Complejo Aljibe; UP 2 - Complejo Tingitania; UP 3 - Complejo
Ronda - Chauen; UP 4 - Complejo Tejeda - Nevada; UP 5 - Complejo Cabo de Gata - Gurugú - Tlemcen.

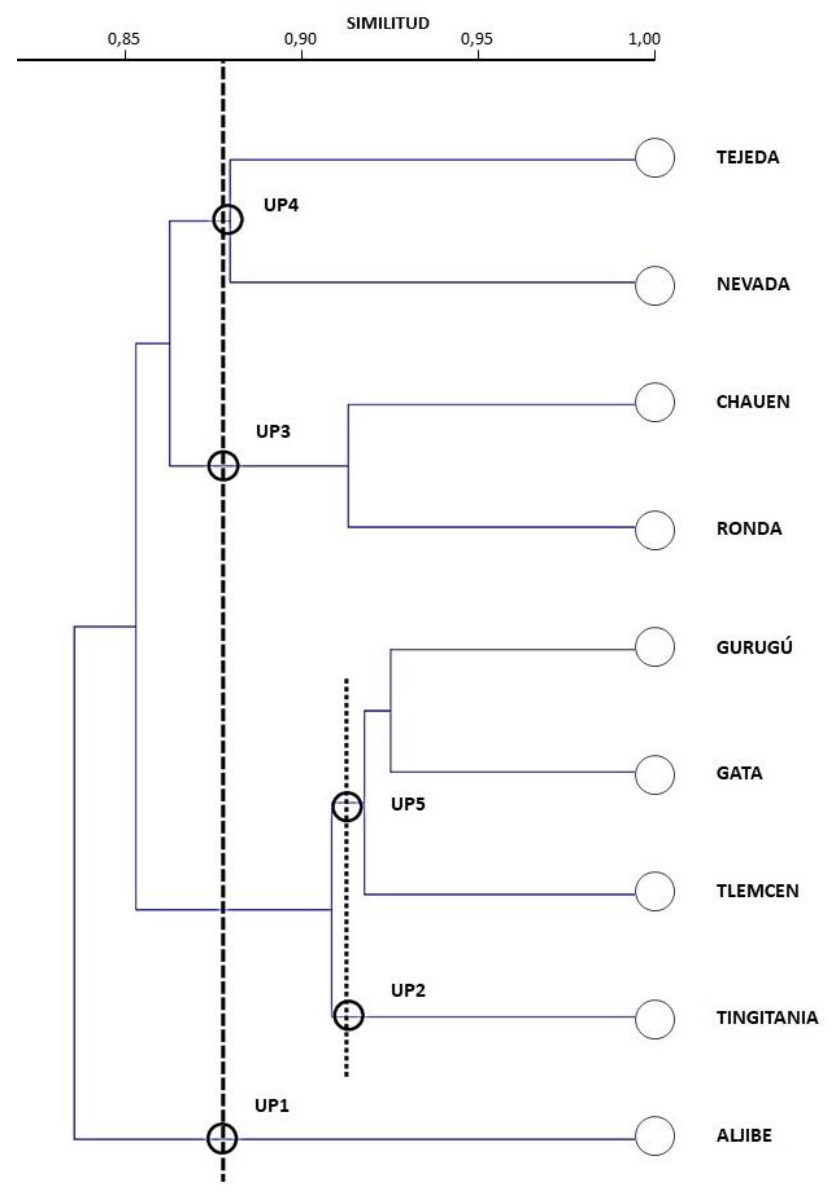

Figura 2. Dendograma de las UGOs en el Arco de Alborán realizado con UPGMA en base a la similitud calculada con el índice de similitud de Bray \& Curtis (Coeficiente de correlación $88,8 \%$; línea de fenon en $88 \%$ de similitud) (UP 1 - Complejo Aljibe; UP 2 - Complejo Tingitania; UP 3 Complejo Ronda - Chauen; UP 4 - Complejo Tejeda Nevada; UP 5 - Complejo Cabo de Gata - Gurugú Tlemcen.

Figure 2. Dendrogram of the OGUs in the Alboran Arc carried out with UPGMA based on the similarity calculated with the similarity index of Bray \& Curtis (Correlation coefficient 88.8\%; Phenon line at 88\%) (PU1: Aljíbica Unit, PU 2: Tingitania Unit, PU 3. Chauen-Ronda Unit, PU 4. Tejeda-Nevada Unit, PU 5. Gurugú-Gata-Tlemcen Unit)

Fronteras y flujos. A partir del cálculo del índice de similitud de Sorensen \& Dice se ha determinado el grado de fortaleza de fronteras pteridogeográficas entre UGOs colindantes y entre UGOs pertenecientes a la misma UP. Entre los pares de UGOs analizados dominan las fronteras pteridogeográficas fuertes, observándose una frontera muy fuerte entre Nevada con Gata. En cuanto a fronteras débiles, sólo se observa una entre Ronda y Chauen y entre Tejeda y Nevada. La determinación de las fronteras junto con la riqueza específica de cada UGO permite la realización del esquema de dirección e intensidad de probabilidad de flujos entre las diferentes UGO (Fig. $4)$. 


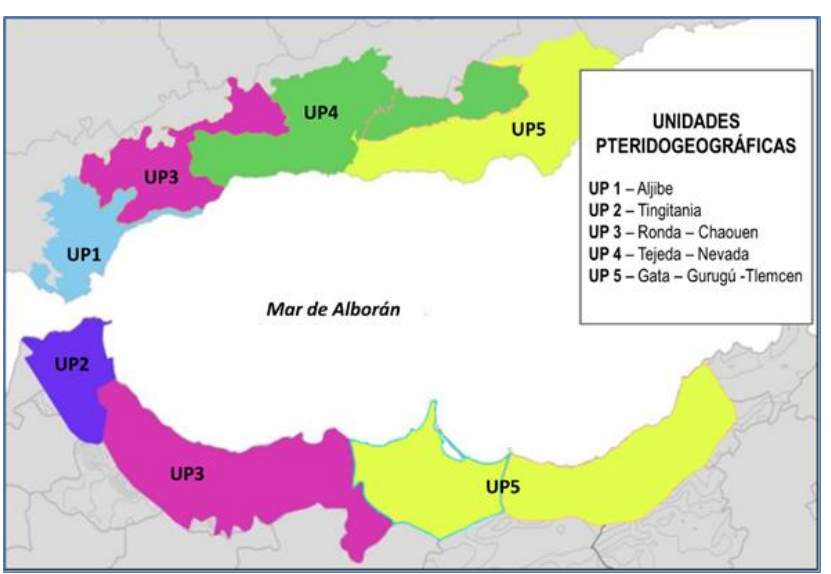

Figura 3. Unidades pteridogeográficas del Arco de Alborán.

Figure 3. Pteridogeographics unities of the Alboran Arc.

En cuanto a los flujos y fronteras, en la vertiente $\mathrm{N}$ se observa una transición florística a lo largo de las cordilleras Béticas desde Aljibe hasta Sierra Nevada. A partir de esta última zona, se observa una frontera muy fuerte que la separa de Cabo de Gata, hecho debido a las grandes diferencias ambientales entre estas UGOs. En Sierra Nevada predomina el clima húmedo en los diversos pisos bioclimáticos, lo cual le confiere una riqueza específica importante en comparación con Cabo de Gata, donde prevalece un clima semiárido. En el caso del Estrecho de Gibraltar, se observa una frontera fuerte entre el Aljibe y la Península Tingitana que, a pesar de tener condiciones ecosistémicas muy similares (Pérez Latorre et al. 1996, 2003), la presión antrópica parece ser un factor determinante en el modelado fitocenótico entre ambos territorios (RodríguezRodríguez et al., 2019) Así, mientras Aljibe disfruta de una mayor riqueza específica gracias a su actual gestión protectora, la falta de una gestión territorial adecuada en Tingitania conlleva a una riqueza específica menor. Igualmente, se identifica una frontera fuerte entre la Península Tingitana y Chauen, con una importante diferencia de valores de riquezas específicas. En este caso también parece influir la presión antrópica, que en Chauen es menos acentuada, y la diferencia de altitud orográfica en estas unidades. Chauen disfruta de mayor número de pisos altitudinales, permitiendo el asentamiento de más diversidad que en Tingitania.

El Macizo del Gurugú se separa de sus unidades geográficas vecinas (Chauen y Tlemcen) mediante fronteras fuertes. El principal causante de la diferencia en ambos casos son sus ombrotipos; así, mientras en Gurugú predomina el seco, los otros dos casos responden a un ombrotipo húmedo.

En cuanto a las relaciones transalboránicas N/S, cabe destacar la frontera débil entre Chauen y Ronda, que pone de manifiesto la alta similitud epiontológica y ecosistémica entre ambas unidades.

\section{Análisis de correspondencia entre variables} ambientales y florísticas

Del análisis de correlación entre las variables ambientales y los índices pteridoflorísticos se deduce:
1) la riqueza específica parece estar determinada por distintas variables, en mayor medida por la duración del período seco y evapotranspiración acumulada durante el mismo periodo cuyo aumento reduce el número de taxones, y de manera menos significativa por la latitud geográfica (disminuye hacia el $\mathrm{N}$ ), la altitud (mayor número cuanto mayor es la altura) y las precipitaciones (aumenta con los valores pluviométricos); 2) la diversidad aumenta a medida que disminuye la longitud, es decir de oeste a este; 3 ) el índice $\mathrm{m} / \mathrm{t}$ aumenta con la altitud y disminuye conforme aumenta la amplitud del periodo seco; y 4) el número cromosómico y el grado de ploidía están determinados de forma positiva por la altitud, y de manera negativa por la ETP, la duración del período seco y la Tmed.

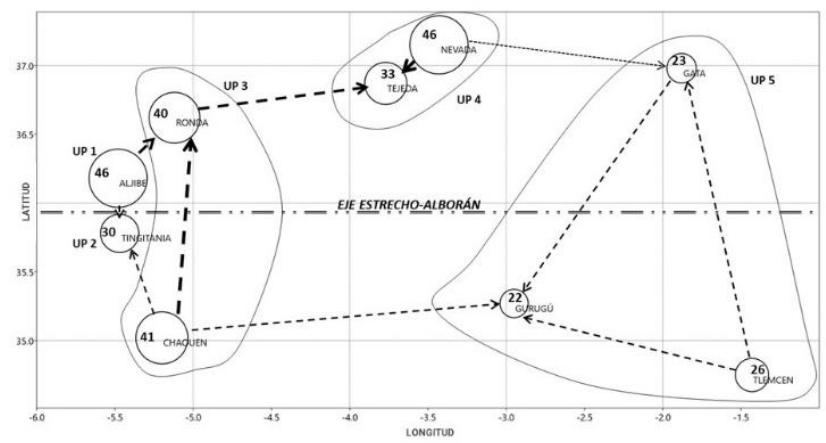

Figura 4. Esquema de probabilidad de dirección de flujo en base a los valores de riqueza pteridofítica de las UGOs e intensidad atendiendo a los grados de frontera (el mayor grosor de las líneas indica una mayor probabilidad de flujo y la flecha su sentido desde UGO de mayor diversidad hacia los de menos).

Figure 4. Flow direction probability diagram based on the pteridophytic wealth values of the UGO and intensity according to the degrees of the border (the mayor thickness of the lines indicates a greater probability of flow and the arrow its direction from UGO of Mayor diversity towards those of less).

\section{Caracterización de Unidades Pteridogeográficas}

En base a los resultados obtenidos sobre la jerarquización de UGOs y establecimiento de unidades pteridogeográficas, constatado por los Análisis de Componentes Principales sobre las variables ambientales e índices pteridofíticos (Fig. 5), se reconocen para el Arco de Alborán 5 unidades pteridogeográficas, cuyos valores de los índices pteridoflorísticos se sintetizan en la Tabla 1.

UP 1: Unidad Aljíbica. Este complejo está compuesto por las Sierras del complejo aljíbico. Esta unidad contiene uno de los mayores valores de riqueza específica, con un total de 46 especies, y un índice de heterogeneidad elevado, índices fuertemente vinculados al ombroclima húmedo de ésta. Por otro lado, le corresponden valores bajos de S/G y $\mathrm{m} / \mathrm{t}$ indicios de la antigüedad de su pteridoflora, como pone de manifiesto el espectro epiontológico. Se observa que el mayor número de relictos paleomediterráneos se localiza en ella, representando un $78,5 \%$ del total de relictos en el Arco de Alborán. Entre las especies relícticas de esta unidad resultan especialmente interesantes Culcita 
macrocarpa C. Presl, Davallia canariensis (L.) Sm., Diplazium caudatum(Cav.) Jermy, Dryopteris guanchica Gibby \& Jermy y Pteris incompleta Cav.

UP 2: Unidad Tingitania. La unidad correspondiente a la unidad de la península tingitana, aunque guarda grandes similitudes en cuanto a las variables ambientales con la unidad anterior, sin embargo, los índices pteridoflorísticos presentan grandes diferencias, teniendo en general los valores más bajos de todo el Arco Alboránico. Un dato relevante es que, a pesar de tener una alta representación de relictos paleomediterráneos, presenta el porcentaje de especies heterocóricas más elevado de toda la región, todo lo cual hace pensar en que, más que problemas derivados de una escasa prospección del territorio, es una consecuencia del fuerte impacto de la ocupación territorial, en especial en los lugares más sensibles para la pteridoflora.
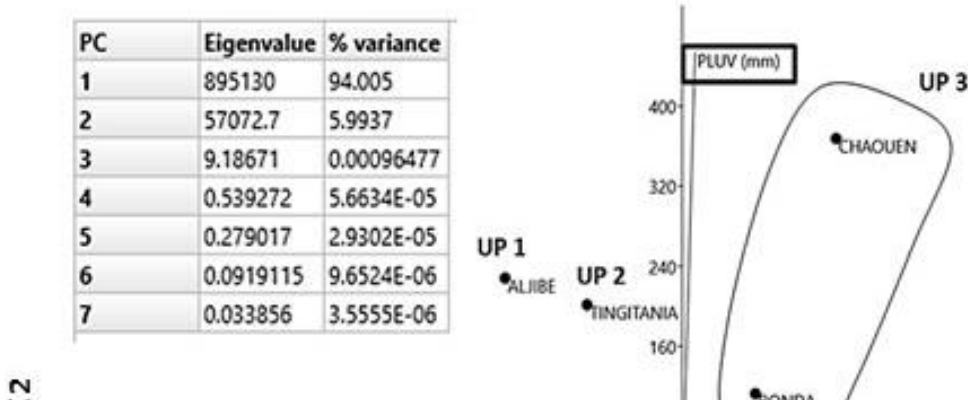

\begin{tabular}{|l|c|c|}
\hline & $P C ~ 1$ & $P C ~ 2$ \\
\hline LONGITUD & 0,000 & 0,005 \\
\hline LATITUD & 0,001 & $-0,002$ \\
\hline ALT max & 0,996 & $-0,091$ \\
\hline Tmed $(\stackrel{\circ}{ }$ C) & $-0,002$ & $-0,002$ \\
\hline Tmin $\left({ }^{\circ} \mathrm{C}\right)$ & $-0,003$ & $-0,002$ \\
\hline OSCT & 0,002 & $-0,002$ \\
\hline PLUV $(\mathrm{mm})$ & 0,091 & 0,996 \\
\hline
\end{tabular}

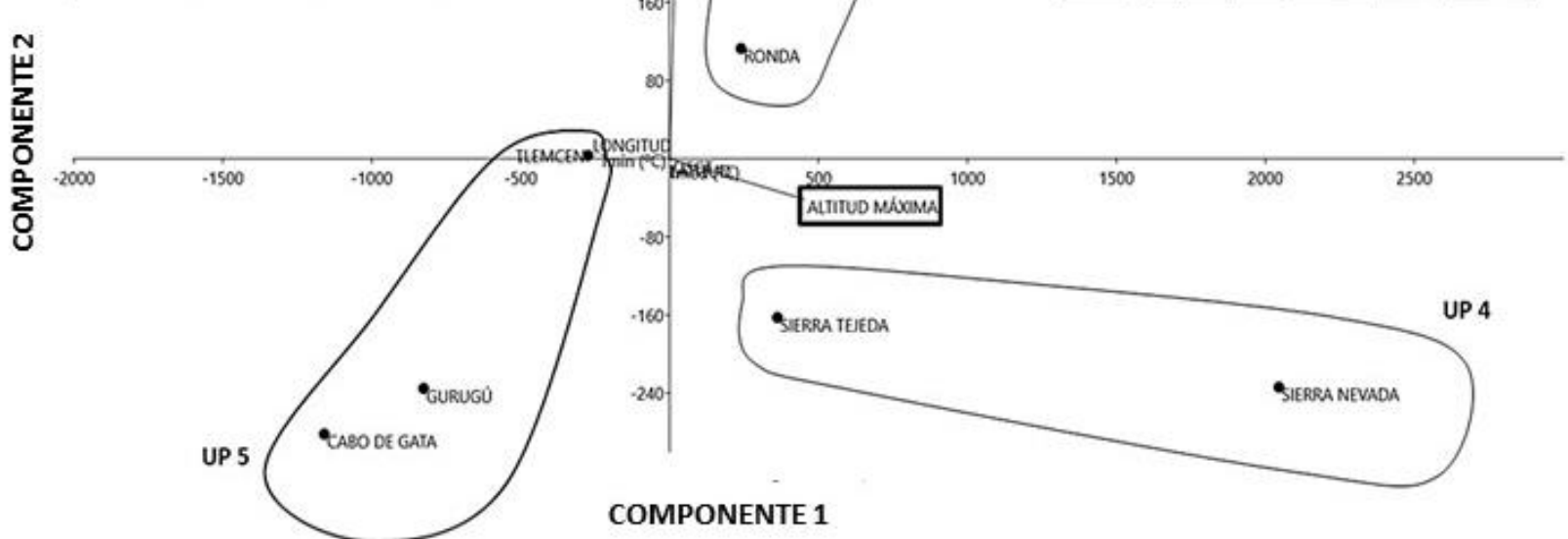

COMPONENTE 1
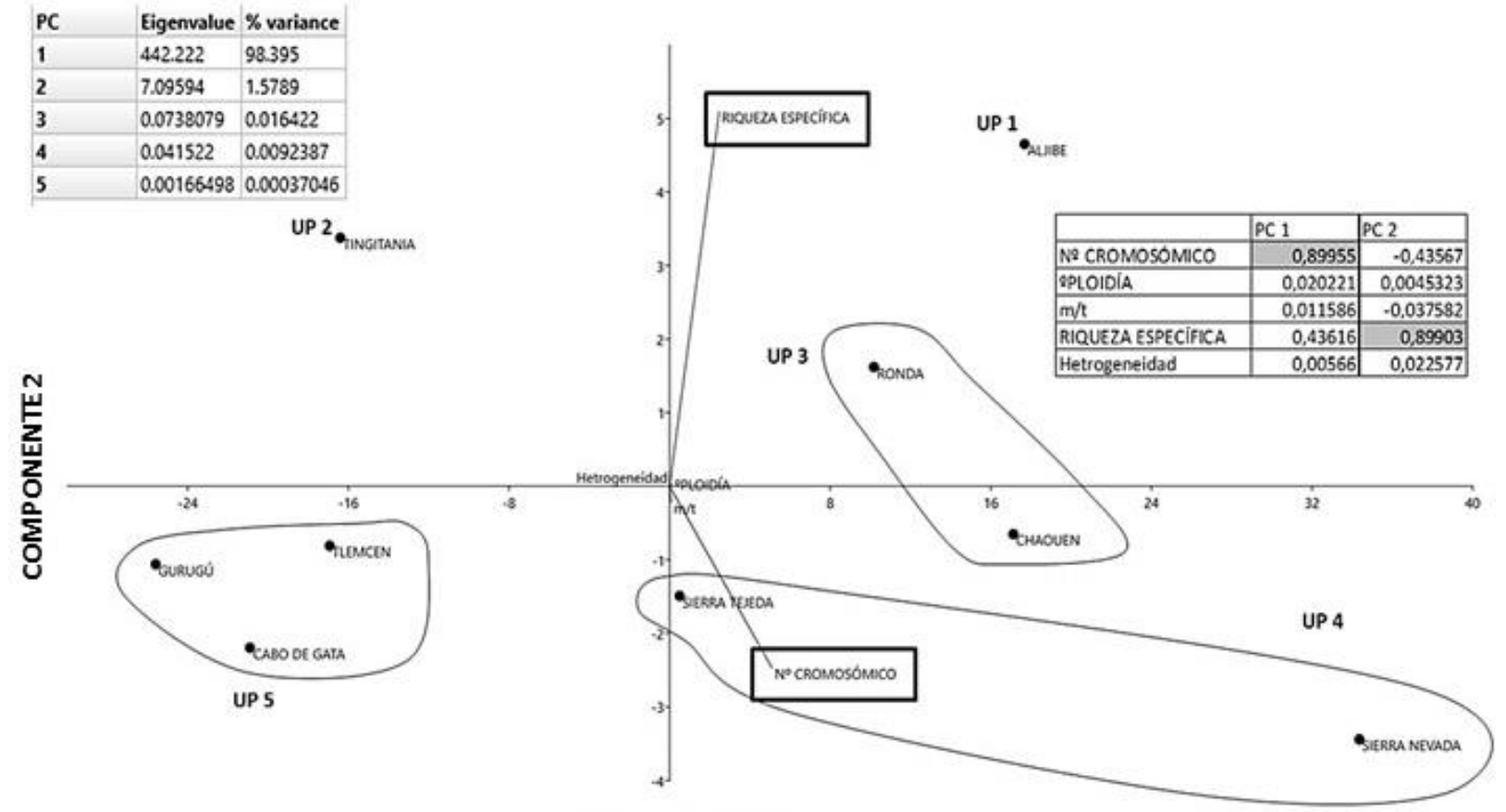

COMPONENTE 1

Figura 5. Análisis de componentes principales de las variables ambientales (arriba) y de los índices pteridoflorísticos (abajo) para las UGOs y su conformación en UPs (en la parte superior izquierda se indica el porcentaje de la varianza de cada una de las tres principales componentes y a la izquierda el peso sobre las dos principales componentes de cada variable, destacando aquellas de mayor peso, que a su vez se destacan en la gráfica).

Figure 5. Principal components analysis of the environmental variables (above) and the pteridofloristic indices (below) for the UGOs and their conformation in PUs (In the upper left part, the percentage of variance of each of the three main components is indicated, and to the left, the weight over the two main components of each variable, highlighting those of greater weight, which in turn are highlighted in the graph). 
Tabla 1. Síntesis de las características pteridogeográficas del Arco de Alborán y de las UPs diferenciadas. (UP1: Unidad Aljíbica, UP 2: Unidad Tingitania, UP 3. Unidad Chauen-Ronda, UP 4. Unidad Tejeda-Nevada, UP 5. Unidad Gurugú-GataTlemcen; ad- Comunidades de fisuras de rocas rezumantes.; ap- Vegetación calcícola y temófila de fisuras de rocas y muros rezumantes; ch- Vegetación silicícola de fisuras de rocas y muros no rezumantes de los pisos inferiores; in- Pastizales húmedos de reducida superficie, compuestos de especies de pequeña talla, rica en terófitos, inundados en invierno y secos en verano; Ic: Vegetación de bosques húmedos y cálidos; ma- Praderas y comunidades de altas hierbas desarrolladas sobre suelos más o menos húmedos (nunca encharcados); na- Pastizales de alta montaña sobre suelos profundos, con hidromorfía temporal por deshielo; oa- Vegetación forestal de borde de río; pc- Vegetación calcícola, no termófila, que coloniza fisuras de rocas y muros no rezumantes; qi- Vegetación de bosques eslerófilos o xerofíticos; qr- Vegetación de bosques caducifolios, mesófilos y oligotrofos; Tr- Comunidades de gleras, calcícolas o basófilas, de origen no fluvial; CIR:Circumboreales; ENMED: Endemismos mediterráneos; HET: Heterocóricas; LAT: Latimediterráneas; MROP: Mediterráneas y de regiones orientales próximas; REPAME: Relictos paleomediterráneos; SUBMED: Submediterráneas; SUBTROP: Subtropicales).

Table 1. Synthesis of the pteridogeographic characteristics of the Alborán Arc and the differentiated PUs. (PU1: Aljibica Unit, PU 2: Tingitania Unit, PU 3. Chauen-Ronda Unit, PU 4. Tejeda-Nevada Unit, PU 5. Gurugú-Gata-Tlemcen Unit); adCommunities of oozing rock fissures .; ap-Calcicolous and thermophilic vegetation of fissures in rocks and oozing walls; chSilicon-rich vegetation of fissures in rocks and non-oozing walls in the lower floors; small-area wet grasslands, composed of species of small size, rich in therophytes, flooded in winter and dry in summer; Ic: Vegetation of humid and warm forests; Ma- prairies and tall grass communities developed on more or less humid soils (never puddled); na- High mountain grasslands on deep soils, with temporary hydromorphy due to thawing; oa- River edge forest vegetation; pc-Calcicolous, non-thermophilic vegetation that colonizes fissures of rocks and non-oozing walls; qi-Vegetation of serophilic or xerophytic forests; qr-Vegetation of deciduous forests ifolios, mesophiles and oligotrophs; Tr-Gleras, calcícolas or basófilas communities, of non-fluvial origin; CIR: Circumboreal; ENMED: Mediterranean endemisms; HET: Heterochoric; LAT: Latimediterranean; MROP: Mediterranean and neighboring eastern regions; REPAME: Paleomediterranean relics; SUBMED: Submediterranean; SUBTROP: Subtropical)

\begin{tabular}{|c|c|c|c|c|c|c|c|}
\hline & & & & & & & \\
\hline & & UP 1 & UP 2 & UP 3 & UP 4 & UP 5 & ARCO ALBORÁNICO \\
\hline \multirow{8}{*}{ 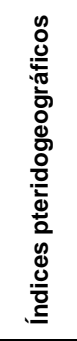 } & Riqueza específica & 46 & 30 & 47 & 47 & 38 & 71 \\
\hline & Rareza acumulada & 43,5 & 26,7 & 43,3 & 44,4 & 35,5 & 67,61 \\
\hline & Rareza media & 0,945 & 0,889 & 0,921 & 0,945 & 0,935 & 0,952 \\
\hline & Heterogeneidad & 3,724 & 3,294 & 3,738 & 3,744 & 3,53 & 4,144 \\
\hline & Ratio S/G & 1,7 & 1,5 & 2,1 & 2,5 & 2,1 & 2,2 \\
\hline & Cociente $\mathrm{m} / \mathrm{t}$ & 1,09 & 1,00 & 1,24 & 1,76 & 1,00 & 1,37 \\
\hline & № cromosómic med & 112,87 & 101,80 & 122,43 & 141,85 & 117,26 & 116,63 \\
\hline & Grado de ploidía & 2,78 & 2,53 & 2,98 & 3,19 & 2,95 & 2,79 \\
\hline \multirow{5}{*}{ 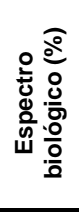 } & Hemicriptófitas & 63,04 & 60,00 & 72,34 & 76,60 & 68,42 & 67,61 \\
\hline & Geófitas & 26,09 & 26,67 & 21,28 & 21,28 & 23,68 & 25,35 \\
\hline & Subcaméfitas & 6,52 & 6,67 & 2,13 & 0,00 & 2,63 & 4,23 \\
\hline & Terófitas & 2,17 & 3,33 & 2,13 & 0,00 & 2,63 & 1,41 \\
\hline & Epífitas & 2,17 & 3,33 & 2,13 & 2,13 & 2,63 & 1,41 \\
\hline \multirow{11}{*}{ 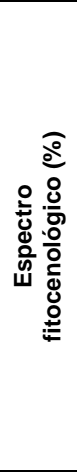 } & ch & 21,74 & 20,00 & 29,79 & 25,53 & 28,95 & 22,54 \\
\hline & oa & 26,09 & 16,67 & 12,77 & 8,51 & 2,63 & 18,31 \\
\hline & ap & 13,04 & 13,33 & 10,64 & 17,02 & 18,42 & 16,90 \\
\hline & in & 10,87 & 13,33 & 10,64 & 6,38 & 10,53 & 11,27 \\
\hline & pc & 6,52 & 6,67 & 10,64 & 10,64 & 10,53 & 7,04 \\
\hline & ad & 8,70 & 13,33 & 8,51 & 6,38 & 10,53 & 5,63 \\
\hline & ma & 4,35 & 6,67 & 6,38 & 8,51 & 7,89 & 5,63 \\
\hline & $q r$ & 4,35 & 3,33 & 6,38 & 8,51 & 5,26 & 5,63 \\
\hline & $\operatorname{tr}$ & 0,00 & 0,00 & 0,00 & 6,38 & 0,00 & 4,23 \\
\hline & Ic & 2,17 & 3,33 & 2,13 & 0,00 & 2,63 & 1,41 \\
\hline & qi & 2,17 & 3,33 & 2,13 & 2,13 & 2,63 & 1,41 \\
\hline \multirow{8}{*}{ 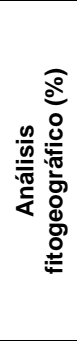 } & CIR & 12,77 & 10,34 & 17,02 & 25,53 & 10,81 & 20,00 \\
\hline & LAT & 19,15 & 27,59 & 19,15 & 23,40 & 21,62 & 20,00 \\
\hline & SUBMED & 21,28 & 13,79 & 17,02 & 19,15 & 24,32 & 17,14 \\
\hline & HET & 14,89 & 20,69 & 19,15 & 17,02 & 18,92 & 12,86 \\
\hline & ENMED & 6,38 & 10,34 & 10,64 & 4,26 & 10,81 & 10,00 \\
\hline & REPAME & 12,77 & 6,90 & 6,38 & 2,13 & 5,41 & 10,00 \\
\hline & \begin{tabular}{|l|} 
MROP \\
\end{tabular} & 6,38 & 6,90 & 8,51 & 6,38 & 5,41 & 5,71 \\
\hline & SUBTROP & 6,38 & 3,45 & 2,13 & 2,13 & 2,70 & 4,29 \\
\hline
\end{tabular}


UP 3. Unidad Chauen-Ronda. Está integrada por Ronda y su correspondiente en la vertiente norteafricana de Chauen. En cuanto a las características biogeográficas presenta los valores más normalizados, representando el carácter global del Arco Alboránico. En cuanto a la epiontología se observa cierta heterogeneidad distribucional entre especies heterocóricas y latemediterráneas, seguidos por especies circumboreales $y$ submediterráneas. Además, cabe destacar el elevado porcentaje de presencia de endemismos mediterráneos $(10,64 \%)$.

UP 4. Unidad Tejeda-Nevada. Biogeográficamente se caracteriza por tener una riqueza específica y una heterogeneidad elevadas, determinadas principalmente por el ombroclima menos xérico que predomina en las cotas más altas. Por otra parte, a la pteridoflora de este complejo biogeográfico le corresponden los valores más elevados de $S / G$ y m/t. Además, en el análisis epiontológico se observa una predominancia de taxones circumboreales y latemediterráneos, en su mayoría postglaciares, que encontraron un refugio en las sierras durante la retirada de las glaciaciones. El conjunto de estos resultados, unido a la casi ausencia de relictos paleomediterráneos, indican que la pteridoflora de la UP 4 es la más moderna. Algunas de las especies más interesante son Asplenium viride Hudson, Cryptogramma crispa (L.) R. Br. ex Hook, Dryopteris tyrrhena Fraser-Jenkins \& Reichst y Gymnocarpium robertianum (Hoffm.) Newman.

UP 5. Unidad Gurugú-Gata-Tlemcen. Este complejo está determinado por su ombroclima árido, lo cual explica su bajo índice de heterogeneidad y su escasa riqueza pteridofítica. A pesar de las condiciones climáticas áridas, la estabilidad climática en el Macizo de Gurugú y Tlemcen permite el crecimiento de diversas especies de helechos que encuentran un refugio gracias a la permanencia de un cinturón nuboso que aporta unos altos niveles de humedad ambiental que se ve incrementada en los barrancos (Escámez, 1989). Desde un punto de vista epiontológico, su pteridoflora está formada principalmente por especies heterocóricas y submediterráneas del Terciario, y algunos relictos paleomediterráneos como Oeosporangium guanchicum (Bolle) Fraser-Jenk. \& Pariyar, Asplenium hemionitis L., y Davallia canariensis (L.) Sm.

\section{Caracterización de corotipos}

A partir de la coincidencia de clústeres tras la repetición 10 veces de 6,8 y 10 centroides mediante el método k-means, se han establecido 11 corotipos. En la Tabla 2 se presenta la síntesis en donde para cada corotipo: se relacionan los taxones que incluye, el porcentaje de presencia de cada corotipo en cada unidad pteridogeográfica, el coeficiente de correlación de cada corotipo respecto a los tracks biogeográficos, y sus índices pteridogeográficos. En consecuencia se observa: a) Los corotipos 8, 9, 10 y 11 responden a un patrón común en todo el Arco de Alborán identificándose su pteridoflora por una importante presencia de especies heterocóricas y de areales mediterráneos expandidos hacia otras regiones vecinas, con un cociente $\mathrm{m} / \mathrm{t}$, número cromosómico medio y grado de ploidía elevados. b) El corotipo 7, integrado por especies endémicas o relictuales, de bajos valores de índices pteridoflorísticos, aunque con presencia en todas las UPs, identifica especialmente a la Unidad Aljíbica. c) Los corotipos 1, 4, 5 y 6 , integrados especialmente por especies circumboreales, distinguen a la Unidad Tejeda-Nevada. d) La Unidad Chauen-Ronda, en su posición de centralidad dentro del Arco Alboránico, se caracteriza por una alta representación de todos los corotipos, con excepción del 1. e) Finalmente la Unidad Gurugú-Gata-Tlemcen queda caracterizada por los corotipos 1,3 y 5 , y la Tingitania por el 2 y 3 .

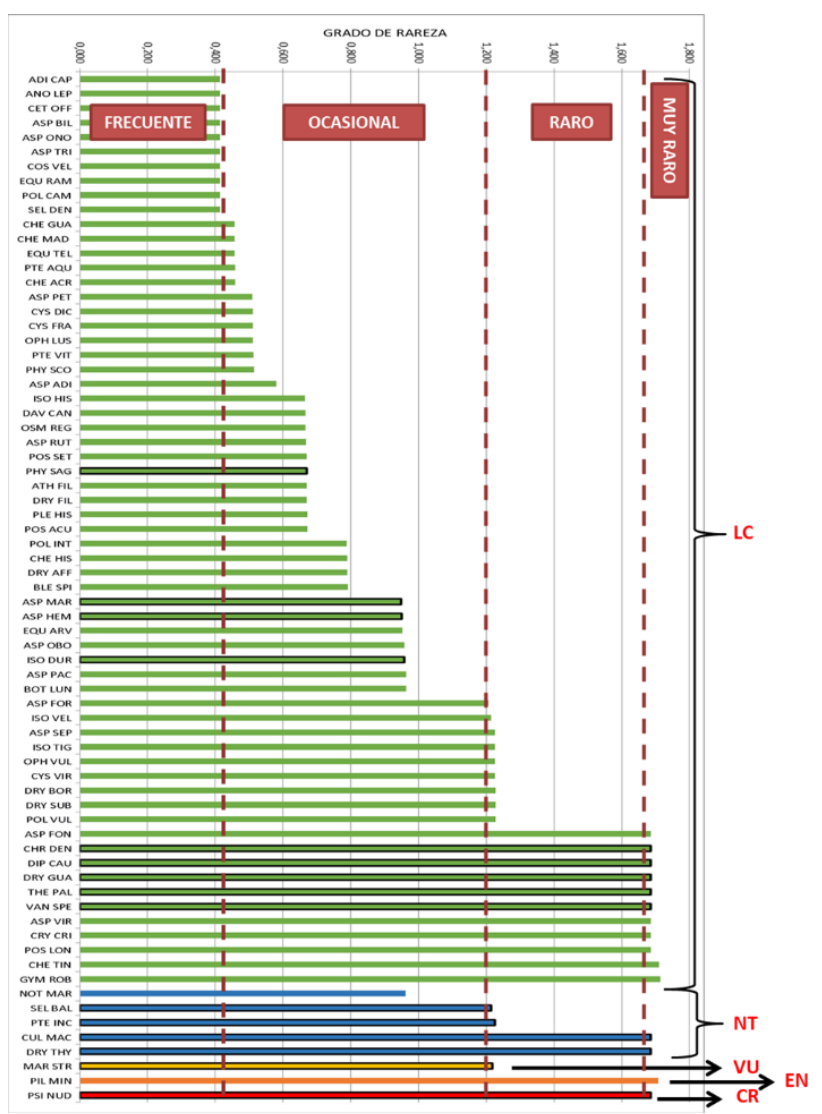

Figura 6. Evaluación de especies amenazadas según la Lista Roja de Licopodios y helechos de UICN (García Criado et al. 2017) respecto al grado de rareza en el Arco de Alborán (LC: preocupación menor, en verde; NT: casi amenazadas, en azul; VU: vulnerable, en amarillo; EN: en peligro, naranja; CR: en peligro crítico, rojo). Las barras bordeadas en negro corresponden a taxones que instituciones regionales, nacionales o internacionales han considerado amenazadas en distintos grados.

Figure 6. Evaluation of threatened species according to the IUCN Red List of Lycopodium and Ferns (García Criado et al. 2017) regarding the degree of rarity in the Alboran Arc (LC: least concern, in green; NT: almost threatened, in blue; VU: vulnerable, in yellow; EN: endangered, orange; $C R$ : critically endangered, red). The bars bordered in black correspond to taxa that regional, national or international institutions have considered threatened to different degrees.

\section{Conservación}

Factores geográficos y epiontológicos convergen en la definición de la pteridoflora del Arco de Alborán presentando una alta diversidad florística de helechos 
y afines (Dudley 2008), si bien más de la mitad de ellos con un alto o muy alto grado de rareza en la región. IUCN ha publicado recientemente una 'Lista Roja de lycopodios y helechos europeos' (García Criado et al., 2017). De los táxones aquí citados el $91 \%$ se consideran como de 'preocupación menor'
(LC), 4 como 'casi amenazadas' (NT, Culcita macrocarpa, Dryopteris tyrrhena, Paragymnopteris marantae y Pteris incompleta), una especie como vulnerable (VU, Marsilea strigosa Willd.), una 'en peligro' (EN, Pilularia minuta Durieu) y una ‘en peligro crítico’ (CR, Psilotum nudum (L.) P. Beauv.). Hasta

Tabla 2. Caracterización de corotipos en el Arco de Alborán. En el primer bloque se relacionan las especies que incluye cada corotipo, a continuación se indican los porcentajes de representación de cada corotipo en cada UP, en el tercer bloque se comparan los corotipos con los tracks biogeográficos, y finalmente se muestran los índices pteridogeográficos para cada corotipo (Las abreviaturas son las de la Tabla 1 y Fig. 6).

Table 2. Characterization of chorotypes in the Alborán Arc. In the first block the species included in each chorotype are listed, then the percentages of representation of each chorotype in each UP are indicated, in the third block the chorotypes are compared with the biogeographic tracks, and finally the pteridogeographic indexes for each chorotype are shown (The abbreviations are those in table 1 and Fig. 6).

\begin{tabular}{|c|c|c|c|c|c|c|c|c|c|c|c|c|}
\hline & & $\begin{array}{c}\text { COROTIPO } \\
1\end{array}$ & $\begin{array}{c}\text { COROTIPO } \\
2\end{array}$ & $\begin{array}{c}\text { COROTIPO } \\
3\end{array}$ & $\begin{array}{c}\text { COROTIPO } \\
4\end{array}$ & $\begin{array}{c}\text { COROTIPO } \\
5\end{array}$ & $\begin{array}{c}\text { COROTIPO } \\
6\end{array}$ & \begin{tabular}{|c|} 
COROTIPO \\
7
\end{tabular} & $\begin{array}{c}\text { COROTIPO } \\
8\end{array}$ & $\begin{array}{c}\text { COROTIPO } \\
9\end{array}$ & $\begin{array}{c}\text { COROTIPO } \\
10\end{array}$ & $\begin{array}{c}\text { COROTIPO } \\
11\end{array}$ \\
\hline & & $\begin{array}{l}\text { ASP FOR } \\
\text { EQU ARV }\end{array}$ & $\begin{array}{c}\text { MAR STR } \\
\text { PIL MIN } \\
\text { ASP OBO }\end{array}$ & $\begin{array}{l}\text { ASP HEM } \\
\text { ASP MAR } \\
\text { SEL BAL }\end{array}$ & $\begin{array}{l}\text { ASP PAC } \\
\text { ASP SEP } \\
\text { ASP VIR } \\
\text { BOT LUN } \\
\text { CRY CRI } \\
\text { CYS VIR } \\
\text { DRY BOR } \\
\text { DRY SUB } \\
\text { DRY THY } \\
\text { OPH VUL } \\
\text { POL VUL } \\
\text { POS LON }\end{array}$ & $\begin{array}{l}\text { CHE HIS } \\
\text { PLE HIS } \\
\text { POL INT } \\
\text { POS ACU }\end{array}$ & $\begin{array}{l}\text { ASP RUT } \\
\text { DRY AFF } \\
\text { DRY FIL }\end{array}$ & $\begin{array}{l}\text { ASP FON } \\
\text { CUL MAC } \\
\text { CHR DEN } \\
\text { DIP CAU } \\
\text { DRY GUA } \\
\text { ISO DUR } \\
\text { ISO TIG } \\
\text { ISO VEL } \\
\text { NOT MAR } \\
\text { PSI NUD } \\
\text { PTE INC } \\
\text { THE PAL } \\
\text { VAN SPE } \\
\text { CHE TIN }\end{array}$ & $\begin{array}{l}\text { PHY SCO } \\
\text { CYS DIC } \\
\text { CYS FRA } \\
\text { PTE AQU }\end{array}$ & $\begin{array}{l}\text { CHE ACR } \\
\text { OPH LUS } \\
\text { PTE VIT }\end{array}$ & $\begin{array}{c}\text { ADI CAP } \\
\text { CHE GUA } \\
\text { CHE MAD } \\
\text { ANO LEP } \\
\text { CET OFF } \\
\text { ASP BIL } \\
\text { ASP ONO } \\
\text { ASP PET } \\
\text { ASP TRI } \\
\text { COS VEL } \\
\text { EQU RAM } \\
\text { EQU TEL } \\
\text { POL CAM } \\
\text { SEL DEN }\end{array}$ & $\begin{array}{c}\text { ISO HIS } \\
\text { ASP ADI } \\
\text { PHY SAG } \\
\text { ATH FIL } \\
\text { POS SET } \\
\text { DAV CAN } \\
\text { OSM REG } \\
\text { BLE SPI }\end{array}$ \\
\hline & UP1 & & & 33,33 & & 25 & 50 & 92,86 & 100 & 100 & 100 & 100 \\
\hline & UP 2 & & 100 & 66,67 & & & & 7,14 & 50 & 100 & 78,57 & 100 \\
\hline & UP 3 & & 33,33 & 66,67 & 41,67 & 100 & 75 & 28,57 & 100 & 100 & 100 & 100 \\
\hline & UP 4 & 100 & & & 100 & 100 & 100 & 7,14 & 100 & 100 & 100 & 37,50 \\
\hline & UP 5 & 100 & 66,67 & 100 & & 75 & 25 & 7,14 & 100 & 100 & 100 & 62,50 \\
\hline & HET & & & & 16,67 & & 66,67 & 7,69 & 50,00 & & 28,57 & 12,50 \\
\hline & CIR & 50,00 & & & 41,67 & & & 15,38 & 25,00 & & 7,14 & 25,00 \\
\hline$\omega \pi$ & SUBTROP & & & & & & & & & 33,33 & & \\
\hline b. & LATEMED & & 50,00 & 33,33 & 25,00 & 50,00 & 33,33 & & 25,00 & & 14,29 & 37,50 \\
\hline 휴 & MROP & & & & 8,33 & & & 7,69 & & 33,33 & 7,14 & \\
\hline 嵅怘 & SUBMED & 50,00 & & & & 25,00 & & 30,77 & & 33,33 & 35,71 & \\
\hline 으 & ENMED & & 50,00 & 33,33 & 8,33 & 25,00 & & 7,69 & & & & 12,50 \\
\hline$\overline{\boldsymbol{n}}$ & REPAME & & & 33,33 & & & & 30,77 & & & 7,14 & 12,50 \\
\hline జు & $\begin{array}{c}\text { № } \\
\begin{array}{c}\text { CROMOSÓMICO } \\
\text { MEDIO }\end{array}\end{array}$ & 180 & 33 & 54 & 158 & 121 & 130 & 79 & 192 & 239 & 113 & 70 \\
\hline 总高玄 & $\begin{array}{c}\text { № MEDIO DE } \\
\text { PLOIDÍA }\end{array}$ & 3,0 & 2,0 & 2,0 & 3,2 & 3,5 & 3,3 & 2,0 & 5,0 & 3,3 & 2,9 & 2,3 \\
\hline & ÍNDICE M/T & 1,00 & 0,00 & 2,00 & 3,00 & 3,00 & 0,00 & 0,63 & 3,00 & 0,00 & 0,75 & 3,00 \\
\hline
\end{tabular}

13 de ellas han sido reconocidas con un grado de amenaza por instituciones regionales, nacionales y/o internacionales. Como se observa en la Fig. 6 de las 12 especies aquí consideradas como muy raras, 8 de ellas son reconocidas con algún status de amenaza por algunas administraciones, y sólo 2 reconoce UICN que requieren un mayor grado de protección. Estos resultados evidencian la escasa efectividad de establecer este tipo de listados para áreas con delimitación política, exentas de criterios biogeográficos, sobre todo para las zonas periféricas de contacto. Resulta paradigmático el caso de Diplazium caudatum (LC en García Criado et al., 2017) que, a pesar de los planes de recuperación, es probablemente uno de los helechos más amenazado de Europa continental.

\section{Conclusiones}

El análisis de correlación entre los índices pteridoflorísticos y las variables ambientales consideradas ponen en evidencia que el gradiente altitudinal y la amplitud del período seco han determinado la distribución de las pteridofitas en el Arco de Alborán. Se observa una distribución en la que predomina el gradiente longitudinal sobre el latitudinal, en el que las pteridofloras de mayor diversidad y más arcaicas se sitúan en el extremo occidental de ambos continentes. Se han diferenciado y caracterizado cinco unidades pteridogeográficas (UP), dos de ellas transalboránicas. Se describen once corotipos en base al grado de presencia en las UP, representación de tracks generalizados e índices pteridoflorísticos. Se ha analizado la situación de preservación de esta pteridoflora en virtud del importante grado de rareza y su escasa consideración en Listas Rojas de reciente publicación, debiendo incorporarse las ocho especies consideradas como raras. Se pone en evidencia la importantísima inclusión de la biogeografía analítica, en especial de un grupo tan especial como el de las pteridofitas, en la gestión del territorio, medio 
ambiente y biodiversidad. Se propone en virtud de los valores de su pteridoflora, atendiendo a su diversidad, antigüedad, rareza y grado de amenaza, la consideración del Arco Alboránico como 'Santuario para los helechos', figura de conservación cada vez más extendida, entendiéndose como una zona de reserva de estas especies para su protección especial.

\section{Agradecimientos}

Deseamos agradecer a la IUCN Centre for Mediterranean Cooperation su colaboración y apoyo, y en especial a su director Antonio Troya Panduro, Andrés Alcántara y Huda Al-Badrawi por su apoyo en las tareas preliminares.

\section{Bibliografía}

Allen, H. (2003). Response of past and present Mediterranean ecosystems to environmental change. Progress in Physical Geography, 27(3), 359-377.

doi: https://doi.org/10.1191/0309133303pp387ra

Badii, M.H., Guillen, A., Pérez, G. \& Aguilar, J. (2015). Extinción de Especies y su Implicación. Species Extinction and its Implication. International Journal of Good Conscience, 10(1), 157-171.

Beals, E.W. (1984). Bray-Curtis ordination: an effective strategy for analysis of multivariate ecological data. Advances in ecological research, 14, 1-55. doi: https://doi.org/10.1016/S0065-2504(08)60168-3

Benabid, A. \& Fennane, M. (1994). Connaissances sur la végétation du Maroc: Phytogéographie, phytosociologie et séries de végétation. Lazaroa, 14, 21-97.

Coll, J., Bourke, D., Gormally, M., Sheehy Skeffington, M. \& Sweeney, J. (2013). Winners and Losers: Climate Change Impacts on Biodiversity in Ireland. Wexford: EPA Climate Change Research Programme 2007-2013. CCRP 19.

Bray, R.J. \& Curtis, J.T. (1957). An ordination of the upland forest communities of southern Wisconsin. Ecological Monographs 27, 325-349. doi: http://dx.doi.org/10.2307/1942268

Cornelissen J.H.C., Lavorel S., Garnier E., Díaz S., Buchmann N., Gurvich D.E., Reich P.B., Steege H. ter, Morgan H.D., Heijden M.G. A. van der, Pausas J.G., \& Poorter H. (2003). A handbook of protocols for standardised and easy measurement of plant functional traits worldwide. Australian Journal of Botany, 51, 335-380. doi: https://doi.org/10.1071/BT02124

Croizat L. (1958). Panbiogeography. Vols. 1 y 2. Caracas: Publicado por el autor.

Della, A.P. \& Falkenberg, D.B. (2019). Pteridophytes as ecological indicators: an overview. Hoehnea, 46, e522018. doi: http://dx.doi.org/10.1590/2236-8906-52/2018.
Dice, L. R. (1945). Measures of the amount of ecologic association between species. Ecology, 26 (3), 297-302.

doi: http://dx.doi.org/10.2307/1932409.

Díez-Garretas, B., \& Salvo-Tierra, Á.E. (1980). Ensayo biogeográfico de los pteridófitos de las Sierras de Algeciras. Anales del Jardín Botánico de Madrid, 37, 455-462. doi: http://dx.doi.org/10.3989/ajbm

Dudley, N. (ed.) (2008). Directrices para la aplicación de las categorías de gestión de áreas protegidas. Gland, Suiza: UICN.

Eşanu, A. (2018). A Backward question about multilevel selection: can species selection help disentangle the notion of group selection?. In C. Jeler (Ed.), Multilevel Selection and the Theory of Evolution (pp. 123-148). London, New York, Shanghai: Palgrave Pivot. doi: https://doi.org/10.1007/978-3-319-78677-3

Escámez Pastrana, A.M. (1989). Los helechos: elementos esenciales en la conservación de nuestra flora. Aldaba, 13, 79-118.

FAO (2006). New_LocClim, Local Climate Estimator Version 1.10. Recurso electrónico en http://www.fao.org/nr/climpag/pub/en0201en.as p. Consulta realizada en enero de 2019.

Fennane M. (2016). Eléments pour un Livre rouge de la flore vasculaire du Maroc. Fasc. 1. Pteridophyta (version 1, nov. 2016). Edit. TelaBotanica. Licence CC-BY NC ND. Recurso electrónico en

file:///C:/Users/Usuario/Downloads/TelaBot_Livr eR-FVM_Fasc-1-nov.2016.pdf.

Consulta realizada en enero de 2019.

Fernández-Badillo, L., Goyenechea, I., \& Escalante, T. (2014). ¿Puede el análisis de endemicidad usarse para la identificación de trazos generalizados? Biogeografía, 7, 15-26.

Flinch, J.F. (1993). Tectonic evolution of the Gibraltar Arc. Houston, TX: Thesis Rice University.

García Criado, M., Väre, H., Nieto, A., Bento Elias, R., Dyer, R., Ivanenko, Y., Ivanova, D., Lansdown, R., Molina, J.A., Rouhan, G., Rumsey, F., Troia, A., Vrba, J. \& Christenhusz, M.J.M. (2017). European Red List of Lycopods and Ferns. Brussels, Belgium: IUCN. doi: http://dx.doi.org/10.2305/IUCN.CH.2017.ERL.1. en

Given, D.R. (1993). Changing Aspects of Endemism and Endangerment in Pteridophyta. Journal of Biogeography, 20 (3), 293-302.

Grant, V. (1981) Plant Speciation. New York, NY: Columbia University Press.

Hammer, Ø. (2019). PAST, PAlaeontological STatistics, Version 3.25. Reference manual. Oslo: University of Oslo. https://folk.uio.no/ohammer/past/past3manual.p df

Haufler, C.H. (2002) Homospory 2002: An Odyssey of Progress in Pteridophyte Genetics and Evolutionary Biology: Ferns and other homosporous vascular plants have highly polyploid chromosome numbers, but they express traits following diploid models and, 
although capable of extreme inbreeding, are predominantly outcrossing, BioScience, (52) 12, 1081-1093. doi: https://doi.org/10.1641/00063568(2002)052[1081:HAOOPI]2.0.CO;2

Hernández-Bermejo, J.E.., \& Sánchez Ollero, $H$. (1983). El análisis de semejanza aplicado al estudio de barreras y fronteras fitogeográficas: su aplicación a la corología y endemoflora ibéricas. Anales del Jardín Botánico de Madrid, 40, 421-435. doi: http://dx.doi.org/10.3989/ajbm

Ito, H. (1972). Distribution of monolete and trilete ferns in eastern Asia and Northern Oceania. The Journal Japanese Botany, 37, 455-462.

Ito, H. (1978). Distribution of two spore patterns in ferns floras of the world (A preliminary survey). The Journal Japanese. Botany, 47, 321-326.

Jaccard, P. (1940). Coefficient generique reel et Coefficient generique probable. Bulletin de la Société Vaudoise des Sciences Naturelles, 61, 117-136.

Jarvinen, O. (1982). Species-to-genus ratios in biogeography: a historical note. Journal of Biogeography, 9(4), 363-370.

doi: https://doi.org/10.2307 / 2844723

Kornas, J. (1985). Adaptive strategies of African pteridophytes to extreme environments. Proceedings of the Royal Society of Edinburgh, 86B, 391-396.

doi: https://doi.org/10.2307 / 2845638

Krug, A.Z., Jablonski, D., \& Valentine, J.W. (2008). Species-genus ratios reflect a global history of diversification and range expansion in marine bivalves. Proceedings of the Royal Society $B$ : Biological Sciences, 275(1639), 1117-1123. doi: https://doi.org/10.1098/rspb.2007.1729

Likas, A., Vlassis, N., \& Verbeek, J.J. (2003). The global k-means clustering algorithm. Pattern Recognition, 36(2), 451-461. IAS-UVA-01-02, 2001, pp.12. ffinria-00321515f https://hal.inria.fr/inria-00321515/document

Maire, R. (1952). Flore de l'Afrique du Nord (Maroc, Algérie, Tunisie, Tripolitaine, Cyrénaïque et Sahara). Paris: Paul Lechevalier.

Márquez, A.L., Real, R., Vargas, J.M., \& Salvo, Á.E. (1997). On identifying common distribution patterns and their causal factors: a probabilistic method applied to pteridophytes in the Iberian Peninsula. Journal of Biogeography, 24(5), 613631.

Medail, F., \& Quézel, P. (1999). Biodiversity hotspots in the Mediterranean Basin: setting global conservation priorities. Conservation tabla. http://dx.doi.org/10.1046/j.15231739.1999.98467.x

Medjahdi, B., Letreuch-Belarouci, A., \& Prelli, P. (2013). Actualisation du catalogue des Ptéridophytes du Nord-Ouest algérien (région de Tlemcen). Acta Botanica Malacitana, 38, 33-39. doi: http://dx.doi.org/10.24310/abm.v38i0.2638

Moreno-Saiz, J.C., Pataro, L., \& Sotomayor, S. P. (2017). Atlas de los pteridófitos de la Península Ibérica e Islas Baleares. Acta Botanica Malacitana, 40, 5-55. doi:http://dx.doi.org/10.24310/abm.v40i0.2540

Nieto, J.M., Cabezudo, B., \& Salvo, Á.E. (1987). Análisis pteridogeográfíco de las sierras Tejeda y Almijara (Málaga-Granada, España). Acta Botanica Malacitana, 12, 103-110.

Oreskes N. (2004). The scientific consensus on climate change. Science, 306, 1686. doi: http://dx.doi.org/10.1126/science. 1103618

Palamarev, E. (1989). Paleobotanical evidences of the Tertiary history and origin of the Mediterranean sclerophyll dendroflora. Plant Systematics and Evolution, 162, 93-107. doi: https://doi.org/10.1007/BF00936912

Palmgren, A. (1925). Die Artenzahl als Pflanzengeographischer Charakter. Acta Bot. Fennica, 49, 1-143.

Pérez Latorre, A.V., Galán de Mera, A., Deil, U. \& Cabezudo, B. (1996). Fitogeografía y vegetación del sector Aljíbico (Cádiz-Málaga, España). Acta Botanica Malacitana 21, 241-267.

Pérez Latorre, A.V., Galán de Mera, A., \& Vicente Orellana, J.A. (2003). Relaciones fitogeográficas entre el suroccidente de la Península Ibérica y el noroeste de África: una propuesta de sectorización. Lagascalia, 23(1), 27-51.

PPG I (2016) A community-derived classification for extant lycophytes and ferns. Journal of Systematics and Evolution, 54, 563-603. doi: https://doi.org/10.1111/jse.12229

Pichi-Sermolli, R. E. G. (1979). A survey of the pteridological flora of the Mediterranean Region. Webbia, 34(1), 175-242.

doi:https://doi.org/10.1080/00837792.1979.1067 0169

Pichi-Sermolli, R.E.G., España L., \& Salvo, A.E. (1988). El valor biogeográfico de la pteridoflora ibérica. Lazaroa, 10, 187-205.

Pla, L. (2006). Biodiversidad: Inferencia basada en el índice de Shannon y la riqueza. Interciencia, 31 (8), 583-590.

Rankou, H., Culham, A., Jury, S.L., \& Christenhusz, M.J. (2013). The endemic flora of Morocco. Phytotaxa, 78(1), 1-69. doi: https://doi.org/10.11646/phytotaxa.78.1.1

Rice, A., Šmarda, P., Novosolov, M., Drori, M., Glick, L., Sabath, N., Meiri, S., Belmaker, J. \& Mayrose, I. (2019). The global biogeography of polyploid plants. Nature Ecology \& Evolution, 3(2), 265.

Rivas-Martínez S., Penas A., Díaz-González T.E., Cantó P., Del Río S., Costa J.C., Herrero L. \& Molero, J. (2017). Biogeographic units of the Iberian Peninsula and Balearic Islands to district level. A concise sinopsis. In Loidi, J. (Ed.), The vegetation of the Iberian Peninsula Vol. 1 (pp. 131-188). doi: https://doi.org/10.1007/978-3-319-54784-8_5

Rivas-Martínez S., Penas A., Díaz-González T.E., Del Río S., Cantó P., Herrero L., Pinto Gomes C. \& Costa J.C. (2014). Biogeography of Spain and Portugal. Preliminary typological sinopsis. International Journal of Geobotanical Research, 4, 1-64. doi: https://doi.org/10.5616/ijgr140001 
Rivas-Martínez, S. (2007). Mapa de series, geoseries y geopermaseries de vegetación de España: [Memoria del mapa de vegetación potencial de España]. Parte I. Itinera Geobotanica, 17, 1-436.

Rivas-Martínez, S. \& Rivas-Sáenz S. (1996-2009). Sistema de Clasificación Bioclimática Mundial. Centro de Investigaciones Fitosociológicas, España. Recurso electrónico en http://www.ucm.es/info/cif. Consulta realizada en enero de 2019.

Rodríguez-Rodríguez, D., Sebastiao, J., Salvo Tierra, Á.E., \& Martínez-Vega, J. (2019). Effect of protected areas in reducing land development across geographic and climate conditions of a rapidly developing country, Spain. Land Degradation \& Development, 30(8), 991-1005. doi: https://doi.org/10.1002/ldr.3286

Sadori, L., Bertini, A., Combourieu-Nebout, N., Kouli, K., Mariotti Lippi, M., Roberts, N., \& Mercuri, A. M. (2013). Palynology and Mediterranean vegetation history. Flora Mediterranea, 23, 141156. doi:https://doi.org/10.7320/FIMedit23.141

Salvo Tierra, Á. E. (1982). Flora peridofítica de Andalucía. Málaga: Tesis Doctoral Universidad de Málaga. https://riuma.uma.es/xmlui/handle/10630/10254

Salvo Tierra, Á. E. (1990). Guía de helechos de la Península lbérica y Baleares. Madrid: Ed. Pirámide.

Salvo, Á.E. (1994). La conservación de la diversidad vegetal en el Campo de Gibraltar: análisis de su pteridoflora como modelo de estrategia de conservación. Almoraima, 11, 195-214.

Salvo, A. E., \& Escaméz, A. (1989). Análisis biogeográfico de la pteridoflora del Macizo del Gurugú (Noreste de Marruecos). Anales del Jardín Botánico de Madrid, 46, 593-598.

Salvo, A. E., \& Garcia-Verdugo, J. C. (1990). Biogeografia numérica en pteridologia.
Monografies de la Societat d'Història Natural de les Balears, 1, 15-149. Palma de Mallorca.

Sluys, R. (1994). Explanations for biogeographic tracks across the Pacific Ocean: a challenge for paleogeography and historical biogeography. Progress in Physical Geography 18(1), 42-58. doi:

https://doi.org/10.1177/030913339401800103

Sørensen, T. (1957). A method of establishing groups of equal amplitude in plant sociology based on similarity of species and its application to analyses of the vegetation on Danish commons». Kongelige Danske Videnskabernes Selskab, 5 (4), 1-34.

Suc, J.P. (1984). Origin and evolution of the Mediterranean vegetation and climate in Europe. Nature, 307, 409-432.

Thompson, J.D. (2005) Plant Evolution in the Mediterranean. Oxford University Press. Oxford. doi:

https://doi.org/10.1093/acprof:oso/97801985153 40.001.0001

Tryon, R.M. \& Tryon A.F. (1982) Ferns and allied plants with special reference to Tropical America. New York: Springer. doi: https://doi.org/110.1007/978-1-4613-8162-4

Valle Tendero, F., Navarro Reyes, F.B., \& Jiménez Morales, M.N. (2004). Datos botánicos aplicados a la Gestión del Medio Natural Andaluz I: bioclimatología y biogeografía. Sevilla: Junta de Andalucía, Consejería de Medio Ambiente.

Varo, J., \& Salvo Tierra, Á.E. (1982). Ensayo biogeográfico sobre la pteridoflora de Sierra Nevada (Granada, España). Acta Botanica Malacitana, 7, 203-210.

Walker, T.G. (1979). The Cytogenetics of ferns. In A. F. Dyer (Ed.), The experimental biology of ferns (pp. 87-732). London, New York: Academic Press. 
ANEXO 1. Variables climáticas de las UGOs.

\begin{tabular}{|c|c|c|c|c|c|c|c|c|c|c|}
\hline \multirow[b]{2}{*}{ UGO } & \multirow[b]{2}{*}{ ESTACIÓN } & \multirow[b]{2}{*}{$\begin{array}{l}\text { LONGITUD } \\
\text { (W) }\end{array}$} & \multirow[b]{2}{*}{$\begin{array}{l}\text { LATITUD } \\
\text { (N) }\end{array}$} & \multirow[b]{2}{*}{$\begin{array}{c}\text { ALTITUD } \\
\text { MÁXIMA (m } \\
\text { s.n.m.) en el } \\
\text { UGO }\end{array}$} & \multicolumn{6}{|c|}{ Variables climáticas } \\
\hline & & & & & $\begin{array}{l}\text { Tmed } \\
\text { (으) }\end{array}$ & $\begin{array}{l}\operatorname{Tmin} \\
\text { (으) }\end{array}$ & $\begin{array}{l}\text { OSCT } \\
\text { (ㄷ) }\end{array}$ & $\begin{array}{l}\text { PLUV } \\
(\mathrm{mm})\end{array}$ & $\begin{array}{l}\text { Long } \\
\text { periodo } \\
\text { seco } \\
\text { (días) } \\
\end{array}$ & $\begin{array}{c}\text { ETP } \\
\text { (mm en } \\
\text { periodo } \\
\text { seco) } \\
\end{array}$ \\
\hline ALJIBE & Los Barrios & 5⒉'W & $36^{\circ}-11^{\prime} \mathrm{N}$ & 1.092 & 17,3 & 8,2 & 11,4 & 803 & 163 & 668,8 \\
\hline CHAUEN & Afeska & 512'W & $35^{\circ} 10^{\prime} \mathrm{N}$ & 2.159 & 13,3 & 0,4 & 16,7 & 1041 & 165 & 827,0 \\
\hline GATA & Carboneras & 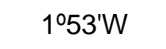 & $36^{\circ} 59^{\prime} \mathrm{N}$ & 562 & 18,2 & 7,6 & 13,7 & 242 & 240 & 966,3 \\
\hline GURUGÚ & Melilla & $2^{\circ} 577^{\prime} \mathrm{W}$ & $35^{\circ}-16^{\prime} \mathrm{N}$ & 890 & 18,4 & 8,3 & 12,2 & 319 & 223 & $1.069,6$ \\
\hline NEVADA & Güéjar Sierra & 3⒉ $26^{\prime} \mathrm{W}$ & $37^{\circ} 9^{\prime} \mathrm{N}$ & 3.479 & 12,7 & $-0,2$ & 18,7 & 583 & 152 & 676,9 \\
\hline RONDA & Igualeja & 50ㄱ'W & $36 \div 37^{\prime} \mathrm{N}$ & 1.919 & 14,9 & 3,9 & 14,8 & 763 & 163 & 668,8 \\
\hline TEJEDA & $\begin{array}{l}\text { Canillas } \\
\text { Aceituno }\end{array}$ & $3^{\circ} 46^{\prime} \mathrm{W}$ & $36^{\circ} 52^{\prime} \mathrm{N}$ & 2.066 & 15,5 & 3,6 & 18,2 & 500 & 152 & 831,5 \\
\hline TINGITANIA & Taghramt & 528'W & $35^{\circ} 47^{\prime} \mathrm{N}$ & 1.362 & 16,9 & 6,3 & 13,5 & 801 & 165 & 827,0 \\
\hline TLEMCEN & Ahfir &  & $34^{\circ}-45^{\prime} \mathrm{N}$ & 1.418 & 13,9 & 1,1 & 18,4 & 607 & 164 & 694,1 \\
\hline
\end{tabular}

ANEXO 2. Catálogo de especies

\begin{tabular}{|c|c|c|c|}
\hline PPG1 & Nomina actualizada & Nomina generalizada & COD \\
\hline \multicolumn{4}{|l|}{ Lycopodiopsida } \\
\hline \multicolumn{4}{|l|}{ Isoëtales } \\
\hline \multicolumn{4}{|c|}{ Isoëtaceae } \\
\hline & Isoëtes durieui Bory & Isoëtes durieui & ISO DUR \\
\hline & Isoëtes histrix Bory & Isoëtes histrix & ISO HIS \\
\hline & Isoëtes tiguliana Gennari & Isoëtes tiguliana & ISO TIG \\
\hline & Isoëtes velata A. Braun & Isoëtes velatum & ISO VEL \\
\hline \multicolumn{4}{|c|}{ Selaginellales } \\
\hline \multicolumn{4}{|c|}{ Selaginellaceae } \\
\hline & Selaginella balansae (A. Braun) Heiron. & Selaginella balansae & SEL BAL \\
\hline & Selaginella denticulata (L.) Spring. & Selaginella denticulata & SEL DEN \\
\hline \multicolumn{4}{|l|}{ Polypodiopsida } \\
\hline \multicolumn{4}{|l|}{ Equisetidae } \\
\hline \multicolumn{4}{|l|}{ Equisetales } \\
\hline \multicolumn{4}{|c|}{ Equisetaceae } \\
\hline & Equisetum arvense $\mathrm{L}$. & Equisetum arvense & EQU ARV \\
\hline & Equisetum ramosissimum Desf. & Equisetum ramosissimum & EQU RAM \\
\hline & Equisetum telmateia Ehrh. & Equisetum telmateia & EQU TEL \\
\hline \multicolumn{4}{|l|}{ Ophiglossidae } \\
\hline \multicolumn{4}{|c|}{ Ophioglossaceae } \\
\hline & Botrychium lunaria (L.) Swatrz & Botrychium lunaria & BOT LUN \\
\hline & Ophioglossum lusitanicum L. & Ophioglossum Iusitanicum & OPH LUS \\
\hline & Ophioglossum vulgatum $\mathrm{L}$. & Ophioglossum vulgatum & OPH VUL \\
\hline
\end{tabular}

Psilotales

Psilotaceae 


\section{Polypodiidae \\ Osmundales}

Osmundaceae

Osmunda regalis $\mathrm{L}$. Osmunda regalis

OSM REG

Hymenophyllales

Hymenophyllaceae

Vandenboschia speciosa (Willd.) Kunkel Vandenboschia speciosa

VAN SPE

Cyatheales

Culcitaceae

Culcita macrocarpa C. Presl Culcita macrocarpa

CUL MAC

Polypodiales

Aspleniaceae

Asplenium adiantum-nigrum $\mathrm{L}$.

Asplenium ceterach L.

Asplenium csikii Kümmerle \& András.

Asplenium fontanum (L.) Bernh.

Asplenium foreziense Magnier

Asplenium hemionitis L.

Asplenium hispanicum (Coss.) Greuter \& Burdet

Asplenium marinum $\mathrm{L}$.

Asplenium obovatum Viv. subsp. obovatum

Asplenium obovatum Viv. subsp. billotii (Schultz) Kerg.

Asplenium onopteris L.

Asplenium petrarchae (Guérin) DC.

Asplenium ruta-muraria $\mathrm{L}$.

Asplenium sagittatum (DC.) Bange

Asplenium scolopendrium L.

Asplenium septentrionale (L.) Hoffm.

Asplenium trichomanes $\mathrm{L}$.

Asplenium viride Hudson

Athyriaceae

Blechnaceae

Athyrium filix-femina (L.) Roth

Diplazium caudatum (Cav.) Jermy

Struthiopteris spicant (L.) F.W. Weiss

Cystopteridaceae

Cystopteris dickieana R. Sim

Cystopteris fragilis (L.) Bernh.

Cystopteris viridula (Desv.) Desv.

Gymnocarpium robertianum (Hoffm.) Newman

Davalliaceae

Polypodiaceae

Davallia canariensis (L.) Sm.

Polypodium cambricum L.

Polypodium interjectum Shivas

Polypodium vulgare L.

Thelypteridaceae

$$
\begin{aligned}
& \text { Thelypteris palustris Schott } \\
& \text { Cyclosorus dentatus (Forssk.) Ching }
\end{aligned}
$$

Dennstaedtiaceae
Asplenium adiantum-nigrum

ASP ADI

Ceterach officinarum

Asplenium pachyrachis

Asplenium fontanum

Asplenium foreziense

Asplenium hemionitis

Pleurosorus hispanicus

Asplenium marinum

Asplenium obovatum

Asplenium billotii

Asplenium onopetris

Asplenium petrachae

Asplenium ruta-muraria

Phyllitis sagittata

Phyllitis scolopendrium

Asplenium septentrionale

Asplenium trichomanes

Asplenium viride

CET OFF

ASP PAC

ASP FON

ASP FOR

ASP HEM

PLE HIS

ASP MAR

ASP OBO

ASP BIL

ASP ONO

ASP PET

ASP RUT

PHY SAG

PHY SCO

ASP SEP

ASP TRI

ASP VIR

Athyrium filix-femina

ATH FIL

Diplazium caudatum

DIP CAU

Blechnum spicant

BLE SPI

Cystopetris dickieana

CYS DIC

Cystopetris fragilis

CYS FRA

Cystopetris viridula

CYS VIR

Gymnocarpium robertianum

GYM ROB

Davallia canariensis

DAV CAN

Polypodium cambricum

POL CAM

Polypodium interjectum

POL INT

Polypodium vulgare

POL VUL

Thelypteris palustris

THE PAL

Christella dentata

CHR DEN

PTE AQU 


\section{Pteridaceae}

Dryopteridacae

\section{Salviniales}

Adiantum capillus-veneris $\mathrm{L}$

Oeosporangium pteridioides (Reichard) Fraser-

Jenk. \& Pariyar subsp. acrosticum (Balb.) Fraser-

Jenk. \& Pariyar

Oeosporangium guanchicum (Bolle) Fraser-Jenk.

\& Pariyar

Oeosporangium hispanicum (Mett.) Fraser-Jenk.

\& Pariyar

Oeosporangium pteridioides (Reichard) Fraser-

Jenk. \& Pariyar subsp. pteridioides

Oeosporangium tinaei (Tod.) Fraser-Jenk.

Anogramma leptophylla (L.) Link

Cosentinia vellea (Aiton) Tod.

Cryptogramma crispa (L.) R. Br. ex Hook.

Paragymnopteris marantae (L.) K.H. Shing

Pteris incompleta Cav.

Pteris vittata $\mathrm{L}$.

Dryopteris affinis (Lowe) Fraser-Jenkins

Dryopteris borreri (Newman) Oberh. \& Tavel

Dryopteris filix-mas (L.) Schott

Dryopteris guanchica Gibby \& Jermy

Dryopteris mindshelkensis N.Pavl.

Dryopteris tyrrhena Fraser-Jenkins \& Reichst.

Polystichum aculeatum (L.) Roth

Polystichum lonchitis (L.) Roth

Polystichum setiferum (Forssk.) Woyn.
Adiantum capillus-veneris

Cheilanthes arcosticha

Cheilanthes guanchica

Cheilanthes hispanica

Cheilantes maderensis

Cheilanthes tinaei

Anogramma leptophyla

Cosentinia vellea

Cryptogramma crispa

Notholaena marantae

Pteris incompleta

Pteris vittata

Dryopteris affinis

Dryopteris borreri

Dryopteris filix-mas

Dryopteris guanchica

Dryopteris submontana

Dryopteris tyrrhena

Polystichum aculeatum

Polystichum lonchitis

Polystichum setiferum
ADI CAP

CHE ACR

CHE GUA

CHE HIS

CHE MAD

CHE TIN

ANO LEP

COS VEL

CRY CRI

NOT MAR

PTE INC

PTE VIT

DRY AFF

DRY BOR

DRY FIL

DRY GUA

DRY SUB

DRY THY

POS ACU

POS LON

POS SET

Marsileaceae

ANEXO 3. Presencia/ausencia de taxones en cada una de las UGOs en el Arco de Alborán.

\begin{tabular}{|c|c|c|c|c|c|c|c|c|c|c|}
\hline & COD & Tingitania & Chauen & Gurugú & Tlemcen & Aljibe & Ronda & Tejeda & Nevada & Gata \\
\hline Adiantum capillus-veneris $\mathrm{L}$ & ADI CAP & 1 & 1 & 1 & 1 & 1 & 1 & 1 & 1 & 1 \\
\hline Anogramma leptophylla (L.) Link & ANO LEP & 1 & 1 & 1 & 1 & 1 & 1 & 1 & 1 & 1 \\
\hline Asplenium adiantum-nigrum $\mathrm{L}$. & ASP ADI & 1 & 1 & 0 & 1 & 1 & 1 & 0 & 1 & 0 \\
\hline Asplenium ceterach $\mathrm{L}$. & CET OFF & 1 & 1 & 1 & 1 & 1 & 1 & 1 & 1 & 1 \\
\hline $\begin{array}{l}\text { Asplenium csikii Kümmerle \& } \\
\text { András. }\end{array}$ & ASP PAC & 0 & 1 & 0 & 0 & 0 & 0 & 1 & 1 & 0 \\
\hline Asplenium fontanum (L.) Bernh. & ASP FON & 0 & 0 & 0 & 0 & 1 & 0 & 0 & 0 & 0 \\
\hline Asplenium foreziense Magnier & ASP FOR & 0 & 0 & 0 & 0 & 0 & 0 & 0 & 1 & 1 \\
\hline Asplenium hemionitis $\mathrm{L}$. & ASP HEM & 1 & 0 & 1 & 1 & 0 & 0 & 0 & 0 & 0 \\
\hline $\begin{array}{l}\text { Asplenium hispanicum (Coss.) } \\
\text { Greuter \& Burdet }\end{array}$ & PLE HIS & 0 & 1 & 0 & 1 & 0 & 1 & 1 & 1 & 0 \\
\hline Asplenium marinum $\mathrm{L}$. & ASP MAR & 1 & 0 & 1 & 0 & 1 & 0 & 0 & 0 & 0 \\
\hline $\begin{array}{l}\text { Asplenium obovatum Viv. subsp. } \\
\text { obovatum }\end{array}$ & ASP OBO & 1 & 1 & 0 & 0 & 0 & 0 & 0 & 0 & 1 \\
\hline $\begin{array}{l}\text { Asplenium obovatum Viv. subsp. } \\
\text { billotii (Schultz) Kerg. }\end{array}$ & ASP BIL & 1 & 1 & 1 & 1 & 1 & 1 & 1 & 1 & 1 \\
\hline Asplenium onopteris L. & ASP ONO & 1 & 1 & 1 & 1 & 1 & 1 & 1 & 1 & 1 \\
\hline $\begin{array}{l}\text { Asplenium petrarchae (Guérin) } \\
\text { DC. }\end{array}$ & ASP PET & 0 & 1 & 1 & 0 & 1 & 1 & 1 & 1 & 1 \\
\hline
\end{tabular}


Asplenium ruta-muraria $\mathrm{L}$.

Asplenium sagittatum (DC.)

Bange

ASP RUT

Asplenium scolopendrium $\mathrm{L}$.

Asplenium septentrionale (L.)

Hoffm.

Asplenium trichomanes L.

Asplenium viride Hudson

Athyrium filix-femina (L.) Roth

Botrychium lunaria (L.) Swatrz

Cosentinia vellea (Aiton) Tod.

Cryptogramma crispa (L.) R.

Br. ex Hook.

Culcita macrocarpa C. Presl

Cyclosorus dentatus (Forssk.)

Ching

Cystopteris dickieana R. Sim

Cystopteris fragilis (L.) Bernh.

Cystopteris viridula (Desv.) Desv.

Davallia canariensis (L.) Sm.

Diplazium caudatum (Cav.) Jermy

Dryopteris affinis (Lowe) Fraser-

Jenkins

Dryopteris borreri (Newman)

Oberh. \& Tavel

Dryopteris filis-mas (L.) Schott

Dryopteris guanchica Gibby \&

Jermy

Dryopteris mindshelkensis N.Pavl.

Dryopteris tyrrhena Fraser-

Jenkins \& Reichst.

Equisetum arvense $\mathrm{L}$.

Equisetum ramosissimum Desf.

Equisetum telmateia Ehrh.

Gymnocarpium robertianum

(Hoffm.) Newman

Isoëtes durieui Bory

Isoëtes histrix Bory

Isoëtes tiguliana Gennari

Isoëtes velata A. Braun

Marsilea strigosa Willd.

$\begin{array}{lllllllll}\text { PHY SAG } & 1 & 1 & 0 & 1 & 1 & 1 & 0 & 0\end{array}$

$\begin{array}{llllllllll}\text { PHY SCO } & 1 & 1 & 0 & 1 & 1 & 1 & 1 & 1 & 0\end{array}$

$\begin{array}{llllllllll}\text { ASP SEP } & 0 & 1 & 0 & 0 & 0 & 0 & 0 & 1 & 0\end{array}$

$\begin{array}{llllllllll}\text { ASP TRI } & 1 & 1 & 1 & 1 & 1 & 1 & 1 & 1 & 1\end{array}$

$\begin{array}{llllllllll}\text { ASP VIR } & 0 & 0 & 0 & 0 & 0 & 0 & 0 & 1 & 0\end{array}$

$\begin{array}{llllllllll}\text { ATH FIL } & 1 & 0 & 0 & 0 & 1 & 1 & 1 & 1 & 0\end{array}$

$\begin{array}{llllllllll}\text { BOT LUN } & 0 & 1 & 0 & 0 & 0 & 0 & 1 & 1 & 0\end{array}$

$\begin{array}{llllllllll}\text { COS VEL } & 1 & 1 & 1 & 1 & 1 & 1 & 1 & 1 & 1\end{array}$

$\begin{array}{llllllllll}\text { CRY CRI } & 0 & 0 & 0 & 0 & 0 & 0 & 0 & 1 & 0\end{array}$

$\begin{array}{llllllllll}\text { CUL MAC } & 0 & 0 & 0 & 0 & 1 & 0 & 0 & 0 & 0\end{array}$

$\begin{array}{llllllllll}\text { CHR DEN } & 0 & 0 & 0 & 0 & 1 & 0 & 0 & 0 & 0\end{array}$

$\begin{array}{llllllllll}\text { CYS DIC } & 0 & 1 & 1 & 1 & 1 & 1 & 1 & 1 & 0\end{array}$

$\begin{array}{llllllllll}\text { CYS FRA } & 0 & 1 & 1 & 1 & 1 & 1 & 1 & 1 & 0\end{array}$

$\begin{array}{llllllllll}\text { CYS VIR } & 0 & 0 & 0 & 0 & 0 & 1 & 0 & 1 & 0\end{array}$

$\begin{array}{llllllllll}\text { DAV CAN } & 1 & 1 & 1 & 0 & 1 & 1 & 0 & 0 & 0\end{array}$

$\begin{array}{llllllllll}\text { DIP CAU } & 0 & 0 & 0 & 0 & 1 & 0 & 0 & 0 & 0\end{array}$

$\begin{array}{llllllllll}\text { DRY AFF } & 0 & 0 & 0 & 0 & 1 & 1 & 1 & 1 & 0\end{array}$

$\begin{array}{llllllllll}\text { DRY BOR } & 0 & 0 & 0 & 0 & 0 & 0 & 1 & 1 & 0\end{array}$

$\begin{array}{llllllllll}\text { DRY FIL } & 0 & 1 & 0 & 0 & 1 & 1 & 1 & 1 & 0\end{array}$

$\begin{array}{llllllllll}\text { DRY GUA } & 0 & 0 & 0 & 0 & 1 & 0 & 0 & 0 & 0\end{array}$

$\begin{array}{llllllllll}\text { DRY SUB } & 0 & 0 & 0 & 0 & 0 & 0 & 1 & 1 & 0\end{array}$

$\begin{array}{llllllllll}\text { DRY THY } & 0 & 0 & 0 & 0 & 0 & 0 & 0 & 1 & 0\end{array}$

$\begin{array}{llllllllll}\text { EQU ARV } & 0 & 0 & 0 & 0 & 0 & 0 & 1 & 1 & 1\end{array}$

$\begin{array}{llllllllll}\text { EQU RAM } & 1 & 1 & 1 & 1 & 1 & 1 & 1 & 1 & 1\end{array}$

$\begin{array}{llllllllll}\text { EQU TEL } & 1 & 1 & 1 & 0 & 1 & 1 & 1 & 1 & 1\end{array}$

$\begin{array}{llllllllll}\text { GYM ROB } & 0 & 0 & 0 & 0 & 0 & 0 & 1 & 0 & 0\end{array}$

$\begin{array}{llllllllll}\text { ISO DUR } & 0 & 0 & 0 & 1 & 1 & 1 & 0 & 0 & 0\end{array}$

$\begin{array}{lllllllllr}\text { ISO HIS } & 1 & 0 & 0 & 1 & 1 & 1 & 0 & 0 & 1\end{array}$

$\begin{array}{llllllllll}\text { ISO TIG } & 0 & 1 & 0 & 0 & 1 & 0 & 0 & 0 & 0\end{array}$

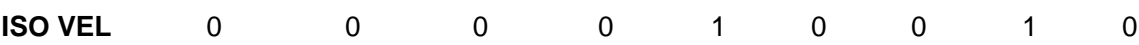

$\begin{array}{rrrrrrrrrr}\text { MAR STR } & 1 & 0 & 0 & 0 & 0 & 0 & 0 & 0 & 1\end{array}$

Oeosporangium pteridioides

(Reichard) Fraser-Jenk. \& Pariyar subsp. acrosticum (Balb.) FraserJenk. \& Pariyar

Oeosporangium guanchicum

(Bolle) Fraser-Jenk. \& Pariyar

Oeosporangium hispanicum

(Mett.) Fraser-Jenk. \& Pariyar

Oeosporangium pteridioides

(Reichard) Fraser-Jenk. \& Pariyar

subsp. pteridioides

Oeosporangium tinaei (Tod.)

Fraser-Jenk.

Ophioglossum lusitanicum L.

Ophioglossum vulgatum L.

Osmunda regalis L.

Paragymnopteris marantae (L.) K.H. Shing

Pilularia minuta Durieu

Polypodium cambricum L.

Polypodium interjectum Shivas

Polypodium vulgare $\mathrm{L}$.

CHE ACR

CHE GUA

CHE HIS

CHE MAD

$\begin{array}{llll}\text { CHE TIN } & 0 & 0\end{array}$

OPH LUS

OPH VUL

OSM REG

NOT MAR

PIL MIN

POL CAM

POL INT

POL VUL

$\begin{array}{ll}1 & 0 \\ 1 & 0 \\ 1 & 1 \\ 1 & 0 \\ 0 & 0 \\ 1 & 1 \\ 1 & 0 \\ 0 & 0\end{array}$


Polystichum aculeatum (L.) Roth

Polystichum lonchitis (L.) Roth

Polystichum setiferum (Forssk.) Woyn.

Psilotum nudum (L.) P. Beauv.

Pteridium aquilinum (L.) Kuhn

Pteris incompleta Cav.

Pteris vittata $\mathrm{L}$.

Selaginella balansae (A. Braun) Heiron.

Selaginella denticulata (L.) Spring. Struthiopteris spicant (L.) F.W. Weiss

Thelypteris palustris Schott Vandenboschia speciosa (Willd.) Kunkel

\begin{tabular}{llllllllll} 
POS ACU & 0 & 1 & 0 & 1 & 0 & 1 & 1 & 1 & 0 \\
POS LON & 0 & 0 & 0 & 0 & 0 & 0 & 0 & 1 & 0 \\
POS SET & 1 & 1 & 0 & 0 & 1 & 1 & 0 & 1 & 0 \\
PSI NUD & 0 & 0 & 0 & 0 & 1 & 0 & 0 & 0 & 0 \\
PTE AQU & 1 & 1 & 1 & 1 & 1 & 1 & 1 & 1 & 0 \\
PTE INC & 1 & 0 & 0 & 0 & 1 & 0 & 0 & 0 & 0 \\
PTE VIT & 1 & 1 & 0 & 0 & 1 & 1 & 1 & 1 & 1 \\
SEL BAL & 0 & 1 & 1 & 0 & 0 & 0 & 0 & 0 & 0 \\
SEL DEN & 1 & 1 & 1 & 1 & 1 & 1 & 1 & 1 & 1 \\
BLE SPI & 1 & 1 & 0 & 0 & 1 & 1 & 0 & 0 & 0 \\
THE PAL & 0 & 0 & 0 & 0 & 1 & 0 & 0 & 0 & 0 \\
VAN SPE & 0 & 0 & 0 & 0 & 1 & 0 & 0 & 0 & 0 \\
\hline
\end{tabular}

ANEXO 4. Cuadro resumen de índices pteridogeográficos de las UGOs en el Arco de Alborán.

\begin{tabular}{cccccccccc}
\hline & Tingitania & Chauen & Gurugú & Tlemcen & Aljibe & Ronda & Tejeda & Nevada & Gata \\
\hline № cromosómico & 102 & 130 & 118 & 121 & 113 & 120 & 130 & 141 & 128 \\
Grado de ploidia & 2,53 & 3,07 & 3,09 & 3,08 & 2,78 & 3,05 & 3,21 & 3,22 & 2,87 \\
Cociente m/t & 1,00 & 1,28 & 1,00 & 1,00 & 1,09 & 1,22 & 1,75 & 1,71 & 0,64 \\
Riqueza específica & 30 & 41 & 22 & 26 & 46 & 40 & 33 & 46 & 23 \\
Riqueza genérica & 18 & 20 & 12 & 12 & 26 & 21 & 16 & 18 & 12 \\
Ratio S/G & 1,67 & 2,05 & 1,83 & 2,17 & 1,77 & 1,90 & 2,06 & 2,56 & 1,92 \\
Coeficiente & 14,40 & 16,20 & 9,54 & 8,51 & 22,11 & 15,98 & 12,56 & 15,72 & 8,21 \\
\hline
\end{tabular}

ANEXO 5. Grado de rareza de los taxones de pteridófitas en el Arco de Alborán.

\begin{tabular}{lccc}
\hline \multicolumn{1}{c}{ ESPECIES } & COD & $\begin{array}{c}\text { MEDIA VALORES } \\
\text { ESTAND. }\end{array}$ & $\begin{array}{c}\text { GRADO DE } \\
\text { RAREZA } \\
(\mathbf{1} / \text { MEDIA) }\end{array}$ \\
\hline Gymnocarpium robertianum (Hoffm.) Newman & GYM ROB & 0,583 & $\mathbf{1 , 7 1}$ \\
Oeosporangium tinaei (Tod.) Fraser-Jenk. & CHE TIN & 0,584 & $\mathbf{1 , 7 1}$ \\
Pilularia minuta Durieu & PIL MIN & 0,585 & $\mathbf{1 , 7 1}$ \\
Asplenium viride Hudson & ASP VIR & 0,593 & $\mathbf{1 , 6 9}$ \\
Cryptogramma crispa (L.) R. Br. ex Hook. & CRY CRI & 0,593 & $\mathbf{1 , 6 9}$ \\
Dryopteris tyrrhena Fraser-Jenkins \& Reichst. & DRY THY & 0,593 & $\mathbf{1 , 6 9}$ \\
Polystichum lonchitis (L.) Roth & POS LON & 0,593 & $\mathbf{1 , 6 9}$ \\
Asplenium fontanum (L.) Bernh. & ASP FON & 0,593 & $\mathbf{1 , 6 9}$ \\
Culcita macrocarpa C. Presl & CUL MAC & 0,593 & $\mathbf{1 , 6 9}$ \\
Cyclosorus dentatus (Forssk.) Ching & CHR DEN & 0,593 & $\mathbf{1 , 6 9}$ \\
Diplazium caudatum (Cav.) Jermy & DIP CAU & 0,593 & $\mathbf{1 , 6 9}$ \\
Dryopteris guanchica Gibby \& Jermy & DRY GUA & 0,593 & $\mathbf{1 , 6 9}$ \\
Psilotum nudum (L.) P. Beauv. & PSI NUD & 0,593 & $\mathbf{1 , 6 9}$ \\
Thelypteris palustris Schott & THE PAL & 0,593 & $\mathbf{1 , 6 9}$ \\
Vandenboschia speciosa (Willd.) Kunkel & VAN SPE & 0,593 & $\mathbf{1 , 6 9}$ \\
Dryopteris borreri (Newman) Oberh. \& Tavel & DRY BOR & 0,814 & $\mathbf{3}$ \\
Dryopteris mindshelkensis N.Pavl. & DRY SUB & 0,814 & $\mathbf{1 , 2 3}$ \\
Polypodium vulgare L. & POL VUL & 0,814 & $\mathbf{1 , 2 3}$ \\
Cystopteris viridula (Desv.) Desv. & CYS VIR & 0,815 & $\mathbf{1 , 2 3}$ \\
Asplenium septentrionale (L.) Hoffm. & ASP SEP & 0,816 & $\mathbf{1 , 2 3}$ \\
Isoëtes tiguliana Gennari & ISO TIG & 0,816 & $\mathbf{1 , 2 3}$ \\
Ophioglossum vulgatum L. & OPH VUL & 0,816 & $\mathbf{1 , 2 3}$ \\
Pteris incompleta Cav. & PTE INC & 0,816 & $\mathbf{1 , 2 3}$ \\
\hline Marsilea strigosa Willd. & MAR STR & 0,821 & $\mathbf{1 , 2 3}$ \\
Selaginella balansae (A. Braun) Heiron. & SEL BAL & 0,824 & $\mathbf{1 , 2 2}$ \\
Isoëtes velata A. Braun & ISO VEL & 0,824 & $\mathbf{1 , 2 1}$ \\
Asplenium foreziense Magnier & ASP FOR & 0,829 & $\mathbf{1 , 2 1}$ \\
Asplenium csikii Kümmerle \& András. & ASP PAC & 1,037 & $\mathbf{0 , 9 6}$ \\
Botrychium lunaria (L.) Swatrz & BOT LUN & 1,037 & $\mathbf{0 , 9 6}$ \\
Paragymnopteris marantae (L.) K.H. Shing & NOT MAR & 1,039 & $\mathbf{0 , 9 6}$
\end{tabular}


A. E. Salvo et al. Biogeografía analítica de la pteridoflora del Arco de Alborán.

Isoëtes durieui Bory

Asplenium obovatum Viv. subsp. obovatum

Equisetum arvense L.

Asplenium hemionitis $\mathrm{L}$.

Asplenium marinum L.

Struthiopteris spicant (L.) F.W. Weiss

Dryopteris affinis (Lowe) Fraser-Jenkins

Oeosporangium hispanicum (Mett.) Fraser-Jenk. \& Pariyar

Polypodium interjectum Shivas

Asplenium hispanicum (Coss.) Greuter \& Burdet

Polystichum aculeatum (L.) Roth

Athyrium filix-femina (L.) Roth

Dryopteris filis-mas (L.) Schott

Asplenium sagittatum (DC.) Bange

Polystichum setiferum (Forssk.) Woyn.

Asplenium ruta-muraria L.

Davallia canariensis (L.) Sm.

Osmunda regalis $\mathrm{L}$.

Isoëtes histrix Bory

Asplenium adiantum-nigrum $\mathrm{L}$.

Asplenium scolopendrium L.

Pteris vittata L.

Ophioglossum lusitanicum L.

Cystopteris dickieana R. Sim

Cystopteris fragilis (L.) Bernh.

Asplenium petrarchae (Guérin) DC.

\begin{tabular}{|c|c|c|c|}
\hline $\begin{array}{l}\text { ISO DUR } \\
\text { ASP OBO } \\
\text { EQU ARV } \\
\text { ASP HEM } \\
\text { ASP MAR } \\
\text { BLE SPI } \\
\text { DRY AFF } \\
\text { CHE HIS } \\
\text { POL INT }\end{array}$ & $\begin{array}{l}1,044 \\
1,044 \\
1,050 \\
1,053 \\
1,055 \\
1,262 \\
1,268 \\
1,268 \\
1,270\end{array}$ & $\begin{array}{l}0,96 \\
0,96 \\
0,95 \\
0,95 \\
0,95 \\
0,79 \\
0,79 \\
0,79 \\
0,79\end{array}$ & \multirow{18}{*}{ 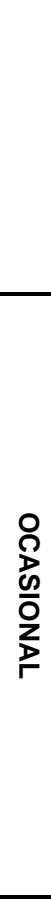 } \\
\hline PLE HIS & 1,489 & 0,67 & \\
\hline POS ACU & 1,489 & 0,67 & \\
\hline ATH FIL & 1,491 & 0,67 & \\
\hline DRY FIL & 1,491 & 0,67 & \\
\hline PHY SAG & 1,491 & 0,67 & \\
\hline POS SET & 1,493 & 0,67 & \\
\hline ASP RUT & 1,496 & 0,67 & \\
\hline DAV CAN & 1,501 & 0,67 & \\
\hline OSM REG & 1,501 & 0,67 & \\
\hline ISO HIS & 1,503 & 0,67 & \\
\hline ASP ADI & 1,722 & 0,58 & \\
\hline PHY SCO & 1,943 & 0,51 & \\
\hline PTE VIT & 1,950 & 0,51 & \\
\hline OPH LUS & 1,958 & 0,51 & \\
\hline CYS DIC & 1,958 & 0,51 & \\
\hline CYS FRA & 1,958 & 0,51 & \\
\hline ASP PET & 1,965 & 0,51 & \\
\hline CHE ACR & 2,179 & 0,46 & \multirow{14}{*}{ 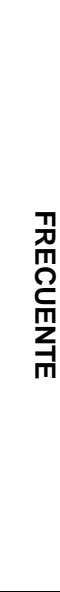 } \\
\hline $\begin{array}{l}\text { PTE AQU } \\
\text { EQU TEL }\end{array}$ & $\begin{array}{l}2,182 \\
2,189\end{array}$ & $\begin{array}{l}0,46 \\
0,46\end{array}$ & \\
\hline CHE GUA & 2,194 & 0,46 & \\
\hline CHE MAD & 2,194 & 0,46 & \\
\hline ADI CAP & 2,418 & 0,41 & \\
\hline ANO LEP & 2,418 & 0,41 & \\
\hline CET OFF & 2,418 & 0,41 & \\
\hline ASP BIL & 2,418 & 0,41 & \\
\hline ASP ONO & 2,418 & 0,41 & \\
\hline ASP TRI & 2,418 & 0,41 & \\
\hline COS VEL & 2,418 & 0,41 & \\
\hline EQU RAM & 2,418 & 0,41 & \\
\hline POL CAM & 2,418 & 0,41 & \\
\hline SEL DEN & 2,418 & 0,41 & \\
\hline
\end{tabular}

Oeosporangium pteridioides (Reichard) Fraser-Jenk. \& Pariyar

subsp. acrosticum (Balb.) Fraser-Jenk. \& Pariyar

Pteridium aquilinum (L.) Kuhn

Equisetum telmateia Ehrh.

Oeosporangium pteridioides (Reichard) Fraser-Jenk. \& Pariyar

subsp. pteridioides

Allosorus pteridioides (Reich.) Christenh.

Adiantum capillus-veneris $\mathrm{L}$.

Anogramma leptophylla (L.) Link

Asplenium ceterach $\mathrm{L}$.

Asplenium obovatum Viv. subsp. billotii (Schultz) Kerg.

Asplenium onopteris L.

Asplenium trichomanes $\mathrm{L}$.

Cosentinia vellea (Aiton) Tod.

Equisetum ramosissimum Desf.

Polypodium cambricum L.

SEL DEN

2,418 